УДК 517.958

\title{
ЗАДАЧА ОБ ОПРЕДЕЛЕНИИ МНОГОМЕРНОГО ЯДРА УРАВНЕНИЯ ВЯЗКОУПРУГОСТИ
}

\author{
Д. К. Дурдиев, Ж. Д. Тотиева
}

\begin{abstract}
Рассматривается интегро-дифференциальная система уравнений вязкоупругости. Прямая задача заключается в определении вектора смещений из начально-краевой задачи для этой системы. Предполагается, что ядро, входящее в интегральный член уравнения, зависит как от временной, так и от пространственной переменной $x_{2}$. Для его отыскания задается дополнительное условие относительно первой компоненты вектора смещения при $x_{3}=0$. Обратная задача заменяется эквивалентной системой интегральных уравнений для неизвестных функций. Исследование проведено на основе метода шкал банаховых пространств аналитических функций. Доказана теорема локальной разрешимости обратной задачи в классе функций, аналитических по переменной $x_{2}$ и непрерывных по $t$.
\end{abstract}

Ключевые слова: обратная задача, устойчивость, дельта-функция, коэффициенты Ламе, ядро.

\section{1. Постановка задачи}

Рассмотрим при $x=\left(x_{1}, x_{2}, x_{3}\right) \in \mathbb{R}^{3}, t \in \mathbb{R}, x_{3}>0$ систему интегро-дифференциальных уравнений

$$
\rho \frac{\partial^{2} u_{i}}{\partial t^{2}}=\sum_{j=1}^{3} \frac{\partial T_{i j}}{\partial x_{j}}, \quad i=1,2,3,
$$

при следующих начальных и граничных условиях:

$$
\begin{gathered}
\left.u_{i}\right|_{t<0} \equiv 0, \\
\left.T_{3 j}\right|_{x_{3}=+0}=-\delta_{1 j} \delta^{\prime}(t) / 2, \quad j=1,2,3,
\end{gathered}
$$

где $u(x, t)=\left(u_{1}(x, t), u_{2}(x, t), u_{3}(x, t)\right)$ - вектор смещений, $\delta^{\prime}(t)-$ производная дельтафункции Дирака; $\delta_{i j}-$ символ Кронекера; $T_{i j}$ - тензор напряжений:

$$
T_{i j}(x, t)=\sigma_{i j}[u](x, t)+\int_{0}^{t} k\left(x_{2}, t-\tau\right) \sigma_{i j}[u](x, \tau) d \tau,
$$

$\sigma_{i j}$ - напряжения, для которых согласно закону Гука имеет место представление

$$
\sigma_{i j}[u](x, t)=\mu\left(\frac{\partial u_{i}}{\partial x_{j}}+\frac{\partial u_{j}}{\partial x_{i}}\right)+\delta_{i j} \lambda \operatorname{div} u .
$$

Система уравнений (1.1)-(1.5) возникает в теории вязкоупругих сред с переменными плотностью $\rho$ и коэффициентами Ламе $\lambda, \mu$. В данной работе предполагается, что $\rho=$

(c) 2015 Дурдиев Д. К., Тотиева Ж. Д. 
$\rho\left(x_{3}\right), \mu=\mu\left(x_{3}\right), \lambda=\lambda\left(x_{3}\right)$ являются функциями одной переменной и принадлежат множеству

$$
\begin{aligned}
\Lambda:=\left\{\rho\left(x_{3}\right), \lambda\left(x_{3}\right), \mu\left(x_{3}\right) \in C^{2}([0, \infty)):\right. & \\
\rho\left(x_{3}\right)>0, \mu\left(x_{3}\right)>0, \lambda\left(x_{3}\right)>0 ; & \left.\rho^{\prime}(+0)=\mu^{\prime}(+0)=\lambda^{\prime}(+0)=0\right\} .
\end{aligned}
$$

При сделанных предположениях из равенств (1.1)-(1.5) следует, что $u_{1}(x, t) \equiv$ $u_{1}\left(x_{2}, x_{3}, t\right) \not \equiv 0, u_{2} \equiv u_{3} \equiv 0$ и функция $\lambda\left(x_{3}\right)$ не будет входить в остающиеся уравнения [1]. Обратная задача заключается в определении ядра $k\left(x_{2}, t\right), t>0$, входящего в (1.1) посредством формулы (1.4), если относительно решения задачи (1.1)-(1.5) известна дополнительная информация

$$
\left.u_{1}\left(x_{2}, x_{3}, t\right)\right|_{x_{3}=+0}=g\left(x_{2}, t\right), \quad t>0,
$$

$g\left(x_{2}, t\right)$ - заданная функция.

Данная постановка обратной задачи без учета зависимости ядра уравнения $k$ от $x_{2}$ была рассмотрена в [2]. При решении применялся принцип сжатых отображений в пространстве непрерывных функций с весовыми нормами к системе интегральных уравнений, которая является эквивалентной обратной задаче. Доказана теорема глобальной однозначной разрешимости и получена оценка устойчивости решения задачи определения ядра. Для исследования обратной задачи поставленной в этой статье используется метод шкал банаховых пространств аналитических функций, развитый в работах Л. В. Овсянникова [3, 4] и Л. Ниренберга [5]. В. Г. Романов с некоторыми модификациями применял этот метод к решению многомерных коэффициентных обратных задач [6-8]. В работах [9-11] на основе этого метода исследованы обратные задачи определения многомерного ядра в гиперболических уравнениях. Здесь вводится шкала аналитических по $x_{2}$ функций и устанавливается локальная теорема существования решения рассматриваемой обратной задачи.

\section{2. Предварительные построения и основной результат}

Определим билинейный интегральный оператор $L$ по формуле

$$
L\left[k\left(x_{2}, t\right), u\left(x_{2}, x_{3}, t\right)\right]=u\left(x_{2}, x_{3}, t\right)+\int_{0}^{t} k\left(x_{2}, t-\tau\right) u\left(x_{2}, x_{3}, \tau\right) d \tau .
$$

В дальнейшем, для сокращения записи, иногда не будем в операторе $L$ указывать зависимость функций от переменных, подразумевая зависимость первой функции от переменных $x_{2}, t$, а второй - от $x_{2}, x_{3}, t$.

Перепишем равенства (1.1)-(1.5) относительно ненулевой компоненты вектора смещений $u_{1}\left(x_{2}, x_{3}, t\right)$ :

$$
\begin{gathered}
\rho\left(x_{3}\right) \frac{\partial^{2} u_{1}}{\partial t^{2}}=\frac{\partial}{\partial x_{3}}\left(\mu\left(x_{3}\right) L\left[k, \frac{\partial u_{1}}{\partial x_{3}}\right]\right)+\mu\left(x_{3}\right) \frac{\partial}{\partial x_{2}}\left(L\left[k, \frac{\partial u_{1}}{\partial x_{2}}\right]\right), \\
\left.u_{1}\right|_{t<0} \equiv 0 \\
L\left[k, \frac{\partial u_{1}}{\partial x_{3}}\right]_{x_{3}=0}=-\frac{\delta^{\prime}(t)}{2 \mu(0)} .
\end{gathered}
$$


Далее индекс «2» у переменной $x$ будет опущен.

Введем в рассмотрение новую переменную $у$ по формуле

$$
y=\phi\left(x_{3}\right):=\int_{0}^{x_{3}} \frac{d \xi}{\nu(\xi)}, \quad \nu\left(x_{3}\right):=\sqrt{\frac{\mu\left(x_{3}\right)}{\rho\left(x_{3}\right)}} .
$$

Через $\phi^{-1}(y)$ обозначим функцию, обратную к $\phi\left(x_{3}\right)$. Пусть

$$
v(x, y, t):=\frac{u_{1}\left(x, \phi^{-1}(y), t\right)}{s(y)}, \quad s(y):=\sqrt{\frac{\nu(+0) \rho(+0)}{\nu\left(\phi^{-1}(y)\right) \rho\left(\phi^{-1}(y)\right)}} .
$$

Тогда обратная задача (2.1)-(2.3), (1.6) в терминах вновь введенных функций и переменной $y$ принимает вид

$$
\begin{gathered}
\frac{\partial^{2} v}{\partial t^{2}}=L\left[k, \frac{\partial^{2} v}{\partial y^{2}}+q(y) v\right]+\nu^{2} \frac{\partial}{\partial x} L\left[k, \frac{\partial v}{\partial x}\right], \quad y>0,(x, t) \in \mathbb{R}^{2}, \\
\left.v(x, y, t)\right|_{t<0} \equiv 0 \\
\frac{\partial}{\partial y} L[k, v]_{y=+0}=-\frac{a \delta^{\prime}(t)}{2}, \\
\left.v(x, y, t)\right|_{y=+0}=g(x, t), \quad t>0, x \in \mathbb{R},
\end{gathered}
$$

где введены обозначения $q(y):=\frac{s^{\prime \prime}(y)}{s(y)}-2\left[\frac{s^{\prime}(y)}{s(y)}\right]^{2}, a=[\mu(+0) \rho(+0)]^{-\frac{1}{2}}$. В уравнении $(2.4)$ и далее под $\nu$ понимается функция $\nu\left(\phi^{-1}(y)\right)$.

Рассмотрим операторное уравнение относительно функции $v(x, y, t)$

$$
L[k, v]=\exp (k(x, 0) t / 2) w(x, y, t) .
$$

Нетрудно проверить, что $v$ через $w$ выражается по формуле

$$
v(x, y, t)=L[r, \exp (k(x, 0) t / 2) w],
$$

где

$$
r(x, t)=-k(x, t)-\int_{0}^{t} k(x, t-\tau) r(x, \tau) d \tau .
$$

Относительно функций $w(x, y, t), v(x, y, t)$ и $r(x, t)$ уравнения $(2.4)-(2.7)$ принимают вид

$$
\begin{gathered}
\frac{\partial^{2} w}{\partial t^{2}}=\frac{\partial^{2} w}{\partial y^{2}}+H(x, y) w-\int_{0}^{t} h(x, t-\tau) w(x, y, \tau) d \tau \\
+\nu^{2} \exp (r(x, 0) t / 2) \frac{\partial}{\partial x} L\left[k, \frac{\partial v}{\partial x}\right], \quad y>0,(x, t) \in \mathbb{R}^{2}, \\
\left.w(x, y, t)\right|_{t<0} \equiv 0, \\
\left.\frac{\partial w}{\partial y}\right|_{y=+0}=-\frac{a \delta^{\prime}(t)}{2}+\frac{a}{4} r(x, 0) \delta(t),
\end{gathered}
$$




$$
\left.w\right|_{y=+0}=L\left[k_{0}, \quad \tilde{g}(x, t)\right],
$$

где

$$
\begin{aligned}
H(x, y) & :=q(y)+\frac{r^{2}(x, 0)}{4}-r_{t}(x, 0), \quad h(x, t):=r_{t t}(x, t) \exp (r(x, 0) t / 2), \\
\tilde{g}(x, t) & :=\frac{a \delta(t)}{2}+\tilde{g}_{0}(x, t) \theta(t), \quad \tilde{g}_{0}(x, t):=g_{0}(x, t) \exp (r(x, 0) t / 2), \\
k_{0}(x, t) & :=k(x, t) \exp (r(x, 0) t / 2), \quad r_{0}(x, t):=r(x, t) \exp (r(x, 0) t / 2) .
\end{aligned}
$$

В последних уравнениях использовано равенство $k(x, 0)=-r(x, 0)$, вытекающее из (2.9). В дальнейшем уравнения (2.10)-(2.13) будем рассматривать в совокупности с равенством (2.8).

Решение прямой задачи $(2.8),(2.10)-(2.12)$ представим в виде

$$
w(x, y, t)=\frac{a}{2} \delta(t-y)+\theta(t-y) \tilde{w}(x, y, t),
$$

где $\tilde{w}(x, y, t)$ - регулярная функция. Тогда, как следует из $(2.8)$, функция $v$ имеет следующий вид:

$$
\begin{gathered}
v(x, y, t)=\frac{a}{2} \exp (k(x, 0) y / 2) \delta(t-y) \\
+\frac{a}{2} r(x, t-y) \exp (k(x, 0) y / 2) \theta(t-y)+\theta(t-y) \tilde{v}(x, y, t),
\end{gathered}
$$

где

$$
\tilde{v}(x, y, t)=L_{0}[r, \exp (k(x, 0) t / 2) \tilde{w}] .
$$

Оператор $L_{0}$ отличается от оператора $L$ лишь тем, что в интеграле в определении $L$ нижний предел равен $y$.

Подставляя функции (2.14) и (2.15) в уравнения (2.10)-(2.13), воспользуемся методом выделения особенностей. При этом прямые вычисления показывают, что последнее слагаемое в (2.10) принимает вид

$$
\begin{gathered}
\frac{\nu^{2} a}{2}\left(k_{x x}(x, 0) \frac{y}{2}+k_{x}^{2}(x, 0) \frac{y^{2}}{4}\right) \delta(t-y) \\
+\frac{\nu^{2} a}{2}\left[\left(k_{x x}(x, 0) \frac{y}{2}+k_{x}^{2}(x, 0) \frac{y^{2}}{4}\right) k(x, t-y)+k_{x}(x, 0) k_{x}(x, t-y)\right] \theta(t-y) \\
+\nu^{2} \exp (r(x, 0) t / 2) \frac{\partial}{\partial x} L_{0}\left[k, \frac{\partial}{\partial x}(r(x, t-y) \exp (k(x, 0) y / 2))+\frac{\partial}{\partial x} \tilde{v}\right] \theta(t-y) .
\end{gathered}
$$

Заметим, что $w=\tilde{w}$ при $t>y>0$. При фиксированном $x \in \mathbb{R}$, обозначая $\beta(x, y):=$ $w(x, y, y)$ и приравнивая коэффициенты при одинаковых особенностях, получим дифференциальное уравнение для нахождения $\beta(x, y)$

$$
\frac{\partial}{\partial y} \beta(x, y)=\frac{a}{4} H(x, y)+\frac{a \nu^{2}}{4}\left(k_{x x}(x, 0) \frac{y}{2}+k_{x}^{2}(x, 0) \frac{y^{2}}{4}\right)
$$

с начальным условием

$$
\beta(x, 0)=-\frac{a}{4} r(x, 0) .
$$

Откуда, найдем

$$
\beta(x, y)=-\frac{a}{4} r(x, 0)+\frac{a \nu^{2}}{4}\left(k_{x x}(x, 0) \frac{y^{2}}{4}+k_{x}^{2}(x, 0) \frac{y^{3}}{12}\right)+\frac{a}{4} \int_{0}^{y} H(x, \xi) d \xi .
$$


Следовательно, функции $w(x, y, t), k(x, t)$ в области $t>y>0$ удовлетворяют уравнениям

$$
\begin{gathered}
\frac{\partial^{2} w}{\partial t^{2}}=\frac{\partial^{2} w}{\partial y^{2}}+H(x, y) w-\frac{a}{2} h(x, t-y)+k_{00}(x, y, t) \\
-\int_{y}^{t} h(x, t-\tau) w(x, y, \tau) d \tau+\nu^{2} \exp (r(x, 0) t / 2) \frac{\partial}{\partial x} L_{0}\left[k, r_{00}+\frac{\partial \tilde{v}}{\partial x}\right], \\
\left.w(x, y, t)\right|_{t=y}=\beta(x, y), \\
\left.\frac{\partial w}{\partial y}\right|_{y=+0}=0, \\
\left.w\right|_{y=+0}=\frac{a}{2} k_{0}(x, t)+L\left[k_{0}, \tilde{g}_{0}\right],
\end{gathered}
$$

где в уравнении (2.17) введены обозначения

$$
\begin{gathered}
k_{00}(x, y, t):=\frac{\nu^{2} a}{2}\left[\left(k_{x x}(x, 0) \frac{y}{2}+k_{x}^{2}(x, 0) \frac{y^{2}}{4}\right) k(x, t-y)+k_{x}(x, 0) k_{x}(x, t-y)\right], \\
r_{00}(x, y, t):=\frac{\partial}{\partial x}(r(x, t-y) \exp (k(x, 0) y / 2)) .
\end{gathered}
$$

Требуя непрерывности функций $w(y, t),\left(\frac{\partial w}{\partial y}\right)(x, y, t)$ при $y=t=0$ из соотношений $(2.18)-(2.20)$ несложно выразить $r(x, 0), r_{t}(x, 0)$ через известные функции:

$$
r(x, 0)=\frac{4}{a} g_{0}(x, 0), \quad r_{t}(x, 0)=-q(0)-\frac{4}{a^{2}} g_{0}^{2}(x, 0)+\frac{4}{a} \tilde{g}_{0 t}(x, 0) .
$$

При выводе последних равенств были использованы следующие равенства:

$$
k_{t}(x, t)=-r_{t}(x, t)-r(x, 0) k(x, t)-\int_{0}^{t} r_{t}(x, t-\tau) k(x, \tau) d \tau, \quad k_{t}(x, 0)=-r_{t}(x, 0)+r^{2}(x, 0) .
$$

В дальнейшем будем считать, что вместо $r(x, 0), r_{t}(x, 0)$ подставлены их значения с помощью формул (2.23), и что они известные функции. Отсюда следует, что функции $k(x, 0)$, $k_{x}(x, 0), k_{x x}(x, 0), k_{t}(x, 0)$, а следовательно, и $k_{00}(x, y, t), r_{00}(x, y, t)$ при $t=y$ являются также известными.

Построим систему интегро-дифференциальных уравнений для функций $w, \tilde{v}, h, k_{0}$. С помощью формулы Даламбера из равенств (2.17), (2.19), (2.20) вытекает уравнение

$$
\begin{gathered}
w(x, y, t)=w_{0}(x, y, t)+\frac{1}{2} \int_{0}^{y} \int_{t-y+\xi}^{t+y-\xi}\left\{H(x, \xi) w(x, \xi, \tau)-\frac{a}{2} h(x, \tau-\xi)\right. \\
+k_{00}(x, \xi, \tau)-\int_{0}^{\tau-\xi} h(x, \gamma) w(x, \xi, \tau-\gamma) d \gamma \\
\left.+\nu^{2} \exp (r(x, 0) \tau / 2) \frac{\partial}{\partial x} L_{0}\left[k, r_{00}+\frac{\partial \tilde{v}}{\partial x}\right](x, \xi, \tau)\right\} d \tau d \xi, \quad x \in \mathbb{R}, 0<y<t
\end{gathered}
$$


где

$$
\begin{gathered}
w_{0}(x, y, t)=\frac{1}{2}\left\{\frac{a}{2}\left(k_{0}(x, t+y)+k_{0}(x, t-y)\right)+\tilde{g}_{0}(x, t+y)\right. \\
\left.+\int_{0}^{t+y} k_{0}(x, \tau) \tilde{g}_{0}(x, t+y-\tau) d \tau+\tilde{g}_{0}(x, t-y)+\int_{0}^{t-y} k_{0}(x, \tau) \tilde{g}_{0}(x, t-y-\tau) d \tau\right\} .
\end{gathered}
$$

Переходя в формуле (2.24) к пределу при $t \rightarrow y+0$, с учетом условий $(2.19),(2.20)$, находим

$$
\begin{aligned}
& 2 \beta(x, y)-w_{0}(x, y, y+0)=\int_{0}^{y} \int_{\xi}^{2 y-\xi}\left\{H(x, \xi) w(x, \xi, \tau)-\frac{a}{2} h(x, \tau-\xi)+k_{00}(x, \xi, \tau)\right. \\
& \left.-\int_{0}^{\tau-\xi} h(x, \gamma) w(x, \xi, \tau-\gamma) d \gamma+\nu^{2} \exp (r(x, 0) \tau / 2) \frac{\partial}{\partial x} L_{0}\left[k, r_{00}+\frac{\partial \tilde{v}}{\partial x}\right](x, \xi, \tau)\right\} d \tau d \xi .
\end{aligned}
$$

Дифференцируя по у последнее равенство, получим:

$$
\begin{gathered}
\frac{\partial \beta}{\partial y}(x, y)-\frac{1}{2} \frac{d w_{0}}{d y}(x, y, y+0)=\int_{0}^{y}\{H(x, \xi) w(x, \xi, 2 y-\xi) \\
-\frac{a}{2} h(x, 2(y-\xi))+k_{00}(x, \xi, 2 y-\xi)-\int_{0}^{2(y-\xi)} h(x, \gamma) w(x, \xi, 2 y-\xi-\gamma) d \gamma \\
\left.+\nu^{2} \exp (r(x, 0)(2 y-\xi) / 2) \frac{\partial}{\partial x} L_{0}\left[k, r_{00}+\frac{\partial \tilde{v}}{\partial x}\right](x, \xi, 2 y-\xi)\right\} d \xi
\end{gathered}
$$

Уравнение для $h$ получается дифференцированием по $y$ равенства (2.25) после замены переменной во втором интеграле $2(y-\xi)$ на $\xi^{\prime}$ :

$$
\begin{gathered}
h(x, y)=h_{0}(x, y)+\frac{2}{a} \int_{0}^{y} k_{0}(x, \xi) \tilde{g}_{0 t t}(x, y-\xi) d \xi+\frac{1}{a} \int_{0}^{y / 2}\left\{H(x, \xi) w_{t}(x, \xi, y-\xi)\right. \\
+k_{00 t}(x, \xi, y-\xi)-\frac{1}{2} h(x, y-2 \xi) \beta(x, \xi)-\int_{0}^{y-2 \xi} h(x, \tau) \frac{\partial w}{\partial t}(x, \xi, y-\xi-\tau) d \tau \\
+\nu^{2} \exp (r(x, 0)(y-\xi) / 2)\left\{\frac{\partial}{\partial x} L_{0}\left[k(x, y-\xi),\left(r_{00 t}+\frac{\partial \tilde{v}_{t}}{\partial x}\right)(x, \xi, y-\xi)\right]\right. \\
+\frac{r(x, 0)}{2} \frac{\partial}{\partial x} L_{0}\left[k(x, y-\xi),\left(r_{00}+\frac{\partial \tilde{v}}{\partial x}\right)(x, \xi, y-\xi)\right] \\
\left.\left.+\frac{1}{2} \frac{\partial}{\partial x}\left[k(x, y-2 \xi)\left(r_{00}+\frac{\partial \tilde{v}}{\partial x}\right)(x, \xi, \xi)\right]\right\}\right\} d \xi,
\end{gathered}
$$


где

$$
\begin{aligned}
h_{0}(x, y) & =k_{0 t t}(x, y)+\frac{2}{a}\left[g_{0}(x, 0) k_{0 t}(x, y)+k_{0}(x, y) \tilde{g}_{0 t}(x, 0)+\tilde{g}_{0 t t}(x, y)\right] \\
- & \frac{2}{a} \frac{\partial^{2}}{\partial y^{2}} \beta(x, y / 2)+\frac{2}{a} H(x, y / 2) \beta(x, y / 2)+\frac{2}{a} k_{00}(x, y / 2, y / 2) \\
& +\frac{2}{a} \nu^{2} \exp (r(x, 0) y / 4)\left(r_{00 x}(x, y / 2, y / 2)+\beta_{x x}(x, y / 2)\right) .
\end{aligned}
$$

Для вычисления функции $w_{t}$ воспользуемся равенством (2.24). Применяя эквивалентное описание области интегрирования в виде $\{(\xi, \tau): t-y \leqslant \tau \leqslant t+y, 0 \leqslant \xi \leqslant$ $y-|t-\tau|\}$, дифференцируем (2.24) по $t$. Вводя замену переменной интегрирования $\tau-t=\xi^{\prime}$, находим:

$$
\begin{gathered}
w_{t}(x, y, t)=w_{0 t}(x, y, t)+\frac{1}{2} \int_{-y}^{y}\{H(x, y-|\xi|) w(x, y-|\xi|, \xi+\tau) \\
-\frac{a}{2} h(x, \xi+t-y+|\xi|)+k_{00}(x, y-|\xi|, t+|\xi|) \\
-\int_{0}^{t-y+\xi+|\xi|} h(x, \gamma) w(x, y-|\xi|, t+\xi-\gamma) d \gamma+\nu^{2} \exp (r(x, 0)(\xi+t) / 2) \\
\left.\times \frac{\partial}{\partial x} L_{0}\left[k(x, \xi+t),\left(r_{00}+\frac{\partial \tilde{v}}{\partial x}\right)(x, y-|\xi|, \xi+t)\right]\right\} \operatorname{sgn}(\xi) d \xi,
\end{gathered}
$$

где

$$
\begin{aligned}
w_{0 t}= & \frac{1}{2}\left\{\frac{a}{2}\left(k_{0 t}(x, t+y)+k_{0 t}(x, t-y)\right)+g_{0}(x, 0)\left[k_{0}(x, t+y)+k_{0}(x, t-y)\right]+\tilde{g}_{0 t}(x, t+y)\right. \\
& \left.+\int_{0}^{t+y} k_{0}(x, \tau) \tilde{g}_{0 t}(x, t+y-\tau) d \tau+\tilde{g}_{0 t}(x, t-y)+\int_{0}^{t-y} k_{0}(x, \tau) \tilde{g}_{0 t}(x, t-y-\tau) d \tau\right\} .
\end{aligned}
$$

Для замыкания системы (2.24), (2.26), (2.27) используем следующие очевидные равенства:

$$
\begin{gathered}
r_{0}(x, t)=r(x, 0)+r_{t}(x, 0) t+\int_{0}^{t}(y-\xi)\left(h(x, \xi)+r(x, 0) r_{0 t}(x, \xi)-\frac{r^{2}(x, 0)}{4} r_{0}(x, \xi)\right) d \xi \\
r_{0 t}(x, t)=r_{t}(x, 0)+\int_{0}^{t}\left(h(x, \xi)+r(x, 0) r_{0 t}(x, \xi)-\frac{r^{2}(x, 0)}{4} r_{0}(x, \xi)\right) d \xi \\
k_{0}(x, t)=-r(x, 0)+\left(\frac{r^{2}(x, 0)}{2}-r_{t}(x, 0)\right) t+\int_{0}^{t}(t-\xi) k_{0 t t}(x, \xi) d \xi \\
k_{0 t}(x, t)=\frac{r^{2}(x, 0)}{2}-r_{t}(x, 0)+\int_{0}^{t} k_{0 t t}(x, \xi) d \xi
\end{gathered}
$$




$$
k_{0 t t}(x, t)=-h(x, t)+\left(\frac{r^{2}(x, 0)}{4}-r_{t}(x, 0)\right) k_{0}(x, t)-\int_{0}^{t} h(x, t-\xi) k_{0}(x, \xi) d \xi,
$$

вытекающие из введенных выше обозначений для $k, r, k_{0}, r_{0}$, а также равенство (2.15), переписанное в виде:

$$
\tilde{v}(x, y, t)=\exp (k(x, 0) t / 2) L_{0}\left[r_{0}, w\right]
$$

которое при дифференцировании по $t$ позволяет вычислить $\tilde{v}_{t}$ :

$$
\tilde{v}_{t}(x, y, t)=\exp (k(x, 0) t / 2)\left(\frac{k(x, 0)}{2} L_{0}\left[r_{0}, w\right]+L_{0}\left[r_{0}, w_{t}\right]+r_{0}(x, t-y) \beta(x, y)\right) .
$$

Пусть $c_{0}(x):=\frac{r^{2}(x, 0)}{4}-r_{t}(x, 0)$. В дальнейшем систему (2.17)-(2.20) будем рассматривать в области

$$
D_{T}=G_{T} \times R, \quad G_{T}=\{(y, t): 0 \leqslant y \leqslant t \leqslant T-y\}, \quad T>0 .
$$

Введем в рассмотрение банахово пространство $A_{s}(r), s>0$, функций $\varphi(x), x \in \mathbb{R}$, аналитических в окрестности начала координат, для которых справедливо соотношение

$$
\|\varphi\|_{s}(r):=\sup _{|x|<r} \sum_{\alpha=0}^{\infty} \frac{s^{\alpha}}{\alpha !}\left\|\frac{\partial^{\alpha}}{\partial x^{\alpha}} \varphi(x)\right\|<\infty, \quad r>0, s>0 .
$$

В дальнейшем параметр $r$ будет считаться фиксированным, в то время как, параметр $s$ рассматривается как переменный параметр. При этом возникает шкала банаховых пространств $A_{s}(r), s>0$. Очевидно следующее свойство: если $\varphi(x) \in A_{s}(r)$, то $\varphi(x) \in A_{s^{\prime}}(r)$ для всех $s^{\prime} \in(0, s)$, следовательно, $A_{s}(r) \subset A_{s^{\prime}}(r)$, если $s^{\prime} \in(0, s)$, и справедливо неравенство

$$
\left\|\frac{\partial^{\alpha}}{\partial x^{\alpha}} \varphi(x)\right\|_{s^{\prime}} \leqslant \alpha^{\alpha} \frac{\|\varphi\|_{s}(r)}{\left(s-s^{\prime}\right)^{\alpha}} \quad(\forall \alpha) .
$$

Так как параметр $r$ фиксирован, будем в дальнейшем опускать его и использовать $\|\varphi\|_{s}, A_{s}$ вместо $\|\varphi\|_{s}(r)$ и $A_{s}(r)$. Через $C_{(y, t)}\left(G_{T} ; A_{s_{0}}\right)$ обозначим класс функций, непрерывных по переменным $(y, t)$ в области $G_{T}$ со значениями в $A_{s_{0}}$. При фиксированных $(y, t)$ норму функции $f(x, y, t)$ в $A_{s_{0}}$ будем обозначать через $\|f\|_{s_{0}}(y, t)$. Норма функции $f$ в $C_{(y, t)}\left(G_{T} ; A_{s_{0}}\right)$ определяется равенством

$$
\|f\|_{C_{(y, t)}\left(G_{T} ; A_{s_{0}}\right)}=\sup _{(y, t) \in G_{T}}\|f\|_{s_{0}}(y, t) .
$$

С целью удобства дальнейших исследований, введем в рассмотрение вектор-функцию $\varphi$ с компонентами

$$
\begin{gathered}
\varphi_{1}(x, y, t)=w(x, y, t)-\frac{1}{2}\left\{\frac{a}{2}\left(k_{0}(x, t+y)+k_{0}(x, t-y)\right)\right. \\
\left.+\int_{0}^{t+y} k_{0}(x, \tau) \tilde{g}_{0}(x, t+y-\tau) d \tau+\int_{0}^{t-y} k_{0}(x, \tau) \tilde{g}_{0}(x, t-y-\tau) d \tau\right\},
\end{gathered}
$$




$$
\begin{gathered}
\varphi_{2}(x, y, t)=\tilde{v}(x, y, t), \\
\varphi_{3}(x, y, t)=w_{t}(x, y, t)-\frac{1}{2}\left\{\frac{a}{2}\left(k_{0 t}(x, t+y)+k_{0 t}(x, t-y)\right)+g_{0}(x, 0)\left[k_{0}(x, t+y)\right.\right. \\
\left.\left.+k_{0}(x, t-y)\right]+\int_{0}^{t+y} k_{0}(x, \tau) \tilde{g}_{0 t}(x, t+y-\tau) d \tau+\int_{0}^{t-y} k_{0}(x, \tau) \tilde{g}_{0 t}(x, t-y-\tau) d \tau\right\}, \\
\varphi_{4}(x, y, t)=\frac{\partial \tilde{v}}{\partial t}(x, y, t), \\
\varphi_{5}(x, y)=2 h(x, y)-c_{0}(x) k_{0}(x, y)-\frac{2}{a}\left[g_{0}(x, 0) k_{0 t}(x, y)+k_{0}(x, y) \tilde{g}_{0 t}(x, 0)\right], \\
\varphi_{6}(x, y)=r_{0}(x, y), \quad \varphi_{7}(x, y)=r_{0 t}(x, y), \quad \varphi_{8}(x, y)=k_{0}(x, y), \quad \varphi_{9}(x, y)=k_{0 t}(x, y) .
\end{gathered}
$$

Пусть

$$
\begin{gathered}
\varphi_{1}^{0}(x, y, t)=\frac{1}{2}\left[\tilde{g}_{0}(x, t+y)+\tilde{g}_{0}(x, t-y)\right], \quad \varphi_{3}^{0}(x, y, t)=\frac{1}{2}\left[\tilde{g}_{0 t}(x, t+y)+\tilde{g}_{0 t}(x, t-y)\right], \\
\varphi_{5}^{0}(x, y)=\frac{2}{a}\left[\tilde{g}_{0 t t}(x, y)-\frac{\partial^{2}}{\partial y^{2}} \beta(x, y / 2)+H(x, y / 2) \beta(x, y / 2)+k_{00}(x, y / 2, y / 2)\right. \\
\left.+\nu^{2} \exp (r(x, 0) y / 4)\left(r_{00 x}(x, y / 2, y / 2)+\beta_{x x}(x, y / 2)\right)\right], \\
\varphi_{6}^{0}(x, y)=r(x, 0)+r_{t}(x, 0) y, \quad \varphi_{7}^{0}(x)=r_{t}(x, 0), \\
\varphi_{8}^{0}(x, y)=-r(x, 0)+\left(\frac{r^{2}(x, 0)}{2}-r_{t}(x, 0)\right) y, \quad \varphi_{9}^{0}(x)=\frac{r^{2}(x, 0)}{2}-r_{t}(x, 0) .
\end{gathered}
$$

Исключая из уравнения (2.26) неизвестную функцию $k_{0 t t}(x, y)$ (эта функция входит в определение $\left.h_{0}\right)$ с помощью $(2.32)$, перепишем систему уравнений $(2.24),(2.26)-(2.34)$ в терминах вектор-функции $\varphi$ :

$$
\begin{gathered}
\varphi_{1}(x, y, t)=\varphi_{1}^{0}(x, y, t)+\frac{1}{2} \int_{0}^{y} \int_{t-y+\xi}^{t+y-\xi}\left\{H(x, \xi) w(x, \xi, \tau)-\frac{a}{2} h(x, \tau-\xi)+k_{00}(x, \xi, \tau)\right. \\
-\int_{0}^{\tau-\xi} h(x, \gamma) w(x, \xi, \tau-\gamma) d \gamma+\nu^{2} \exp (r(x, 0) \tau / 2) \\
\left.\times \frac{\partial}{\partial x} L_{0}\left[\varphi_{8}(x, \tau) \exp (-r(x, 0)(\tau) / 2),\left(r_{00}+\frac{\partial \varphi_{2}}{\partial x}\right)(x, \xi, \tau)\right]\right\} d \tau d \xi \\
-\frac{a}{2} h(x, \xi+t-y+|\xi|)+k_{00}(x, y-|\xi|, t+|\xi|) \\
\varphi_{3}(x, y, t)=\varphi_{3}^{0}+\frac{1}{2} \int_{-y}^{y}\{H(x, y-|\xi|) w(x, y-|\xi|, \xi+\tau) \\
\quad \int_{0}^{t-y+\xi+|\xi|} h(x, \gamma) w\left(x, y-|\xi|, t_{\xi}-\gamma\right) d \gamma+\nu^{2} \exp (r(x, 0)(\xi+t) / 2) \frac{\partial}{\partial x} L_{0}
\end{gathered}
$$




$$
\begin{gathered}
\varphi_{5}(x, y)=\varphi_{5}^{0}(x, y)+\int_{0}^{y}\left(\frac{2}{a} \tilde{g}_{0 t t}(x, y-\xi)-h(x, y-\xi)\right) \varphi_{8}(x, \xi) d \xi \\
+\frac{1}{a} \int_{0}^{y / 2}\left\{H(x, \xi) w_{t}(x, \xi, y-\xi)+k_{00 t}(x, \xi, y-\xi)-\frac{1}{2} h(x, y-2 \xi) \beta(x, \xi)\right. \\
-\int_{0}^{y-2 \xi} h(x, \tau) w_{t}(x, \xi, y-\xi-\tau) d \tau+\nu^{2} \exp (r(x, 0)(y-\xi) / 2) \\
\times\left\{\frac{\partial}{\partial x} L_{0}\left[\varphi_{8}(x, y-\xi) \exp (-r(x, 0)(y-\xi) / 2),\left(r_{00 t}+\frac{\partial \varphi_{4}}{\partial x}\right)(x, \xi, y-\xi)\right]\right. \\
+\frac{r(x, 0)}{2} \frac{\partial}{\partial x} L_{0}\left[\varphi_{8}(x, y-\xi) \exp (-r(x, 0)(y-\xi) / 2),\left(r_{00}+\frac{\partial \varphi_{2}}{\partial x}\right)(x, \xi, y-\xi)\right] \\
\left.\left.+\frac{1}{2} \frac{\partial}{\partial x}\left[\varphi_{8}(x, y-2 \xi) \exp (-r(x, 0)(y-2 \xi) / 2)\left(r_{00}+\frac{\partial \varphi_{2}}{\partial x}\right)(x, \xi, \xi)\right)\right]\right\} d \xi \\
\varphi_{6}(x, y)=\varphi_{6}^{0}(x, y)+\int_{0}^{y}(y-\xi)\left(h(x, \xi)+r(x, 0) \varphi_{7}(x, \xi)-\frac{r^{2}(x, 0)}{4} \varphi_{6}(x, \xi)\right) d \xi \\
\varphi_{8}(x, y)=\varphi_{8}^{0}(x, y)+\varphi_{9}^{0}(x) t+\int_{0}^{y}(y-\xi)\left(c_{0}(x) \varphi_{8}(x, \xi)-L\left[\varphi_{8}, h\right]\right)(x, \xi) d \xi \\
\varphi_{7}(x, y)=\varphi_{7}^{0}(x)+\int_{0}^{y}\left(h(x, \xi)+r(x, 0) \varphi_{7}(x, \xi)-\frac{r^{2}(x, 0)}{4} \varphi_{6}(x, \xi)\right) d \xi
\end{gathered}
$$

где вместо функций $\varphi_{2}, \varphi_{4}$ подразумеваются их соответствующие представления (2.33)$(2.34)$, a

$$
\begin{aligned}
w(x, y, t) & =\varphi_{1}(x, y, t)+\frac{1}{2}\left\{\frac{a}{2}\left(\varphi_{8}(x, t+y)+\varphi_{8}(x, t-y)\right)\right. \\
& \left.+\int_{0}^{t+y} \varphi_{8}(x, \tau) \tilde{g}_{0}(x, t+y-\tau) d \tau+\int_{0}^{t-y} \varphi_{8}(x, \tau) \tilde{g}_{0}(x, t-y-\tau) d \tau\right\} \\
w_{t}(x, y, t) & =\varphi_{3}(x, y, t)+\frac{1}{2}\left\{\frac{a}{2}\left(\varphi_{9}(x, t+y)+\varphi_{9}(x, t-y)\right)+g_{0}(x, 0)\left[\varphi_{8}(x, t+y)\right.\right. \\
& \left.\left.+\varphi_{8}(x, t-y)\right]+\int_{0}^{t+y} \varphi_{8}(x, \tau) \tilde{g}_{0 t}(x, t+y-\tau) d \tau+\int_{0}^{t-y} \varphi_{8}(x, \tau) \tilde{g}_{0 t}(x, t-y-\tau) d \tau\right\} \\
h(x, y)= & \frac{1}{2}\left[\varphi_{5}(x, y)+c_{0}(x) \varphi_{8}(x, y)\right]+\frac{1}{a}\left[g_{0}(x, 0) \varphi_{9}(x, y)+\varphi_{8}(x, y) \tilde{g}_{0 t}(x, 0)\right] .
\end{aligned}
$$


Основной результат статьи составляет теорема локальной однозначной разрешимости в классе функций, аналитических по переменной $x_{2}$.

Теорема. Пусть $(\rho, \mu, \lambda) \in \Lambda$, кроме того

$$
\begin{gathered}
\left(\tilde{g}_{0}(x, 0), \tilde{g}_{0 t}(x, 0)\right) \in A_{s_{0}}, \\
\left(\tilde{g}_{0}(x, t), \tilde{g}_{0 t}(x, t), \tilde{g}_{0 t t}(x, t)\right) \in C_{t}\left([0, T] ; A_{s_{0}}\right), \\
\max \left\{\left\|\tilde{g}_{0}\right\|_{s_{0}}(t),\left\|\tilde{g}_{0 t}\right\|_{s_{0}}(t),\left\|\tilde{g}_{0 t t}\right\|_{s_{0}}\right\} \leqslant R, \\
\max \left\{\|\beta\|_{s_{0}}(y),\|H\|_{s_{0}}(y), \max \left(1, \nu_{0}^{2}\right)\|\exp ( \pm r(x, 0) t / 2)\|_{s_{0}}(t)\right\} \leqslant R, \\
\max \left\{\left\|\varphi_{i}^{0}\right\|_{s_{0}}(t)\right\} \leqslant R, \quad i=5,6,7,8,9, \\
\nu_{0}^{2}=\max _{0 \leqslant y \leqslant \frac{T}{2}} \nu^{2}\left(\phi^{-1}(y)\right), \quad t \in[0, T], y \in[0, T / 2], R>0 .
\end{gathered}
$$

Тогда найдется такое $b \in(0, T / 2)$, что для любого $s \in\left(0, s_{0}\right)$ в области $\Gamma_{s T}=$ $D_{T} \cap\left\{(x, y, t): 0 \leqslant y \leqslant b\left(s_{0}-s\right)\right\}$ существует единственное решение системы уравнений (2.36)-(2.42), для которого

$$
\begin{gathered}
\varphi_{1}(x, y, t), \varphi_{3}(x, y, t) \in C_{(y, t)}\left(P_{s T} ; A_{s_{0}}\right), \\
\varphi_{i}(x, t) \in C_{t}\left(\left[0, b\left(s_{0}-s\right)\right] ; A_{s_{0}}\right), \quad i=5,6,7,8,9, \\
P_{s T}=G_{T} \cap\left\{(y, t) \mid 0 \leqslant y \leqslant b\left(s_{0}-s\right)\right\},
\end{gathered}
$$

причем

$$
\begin{gathered}
\left\|\varphi_{i}-\varphi_{i}^{0}\right\|_{s} \leqslant R, i=1,6,8, \quad\left\|\varphi_{i}-\varphi_{i}^{0}\right\|_{s} \leqslant \frac{R}{s_{0}-s}, i=3,7,9 \\
\left\|\varphi_{5}-\varphi_{5}^{0}\right\|_{s} \leqslant \frac{R}{\left(s_{0}-s\right)^{2}}, \quad(y, t) \in P_{s T}
\end{gathered}
$$

\section{3. Доказательство теоремы}

В условиях теоремы

$$
\varphi_{i}^{0} \in C_{(y, t)}\left(G_{T} ; A_{s_{0}}\right), \quad\left\|\varphi_{i}^{0}\right\|_{s}(y, t) \leqslant R, \quad i=1,3, \quad(y, t) \in G_{T}, 0<s<s_{0} .
$$

Пусть $b_{n}$ являются членами монотонно убывающей последовательности, определяемой равенствами

$$
b_{n+1}=\frac{b_{n}}{1+1 /(n+1)^{2}}, \quad n=0,1,2, \ldots
$$

Обозначим

$$
b=\lim _{n \rightarrow \infty} b_{n}=b_{0} \prod_{n=0}^{\infty}\left(1+1 /\left(n+1^{2}\right)\right)^{-1} .
$$

Число $b_{0} \in(0, T / 2)$ будет выбрано подходящим образом. 
Построим процесс последовательных приближений по схеме

$$
\begin{aligned}
& \varphi_{1}^{n+1}(x, y, t)=\varphi_{1}^{0}(x, y, t)+\frac{1}{2} \int_{0}^{y} \int_{t-y+\xi}^{t+y-\xi}\left\{H(x, \xi) w^{n}(x, \xi, \tau)-\frac{a}{2} h^{n}(x, \tau-\xi)+k_{00}^{n}(x, \xi, \tau)\right. \\
& -\int_{0}^{\tau-\xi} h^{n}(x, \gamma) w^{n}(x, \xi, \tau-\gamma) d \gamma+\nu^{2} \exp (r(x, 0) \tau / 2) \\
& \left.\times \frac{\partial}{\partial x} L_{0}\left[\varphi_{8}^{n}(x, \tau) \exp (-r(x, 0)(\tau) / 2),\left(r_{00}^{n}+\frac{\partial \varphi_{2}^{n}}{\partial x}\right)(x, \xi, \tau)\right]\right\} d \tau d \xi, \\
& \varphi_{3}^{n+1}(x, y, t)=\varphi_{3}^{0}(x, y, t)+\frac{1}{2} \int_{-y}^{y}\left\{H(x, y-|\xi|) w^{n}(x, y-|\xi|, \xi+t)-\frac{a}{2} h^{n}(x, \xi+t-y+|\xi|)\right. \\
& +k_{00}^{n}(x, y-|\xi|, t+|\xi|)-\int_{0}^{t-y+\xi+|\xi|} h^{n}(x, \gamma) w^{n}(x, y-|\xi|, t+\xi-\gamma) d \gamma \\
& +\nu^{2} \exp (r(x, 0)(\xi+t) / 2) \frac{\partial}{\partial x} L_{0}\left[\varphi_{8}^{n}(x, \xi+t)\right. \\
& \left.\left.\times \exp (-r(x, 0)(\xi+t) / 2),\left(r_{00}^{n}+\frac{\partial \varphi_{2}^{n}}{\partial x}\right)(x, y-|\xi|, \xi+t)\right]\right\} \operatorname{sgn}(\xi) d \xi, \\
& \varphi_{5}^{n+1}(x, y)=\varphi_{5}^{0}(x, y)+\int_{0}^{y}\left(\frac{2}{a} \tilde{g}_{0 t t}(x, y-\xi)-h^{n}(x, y-\xi)\right) \varphi_{8}^{n}(x, \xi) d \xi \\
& +\frac{1}{a} \int_{0}^{y / 2}\left\{H(x, \xi) w_{t}^{n}(x, \xi, y-\xi)+k_{00 t}^{n}(x, \xi, y-\xi)-\frac{1}{2} h^{n}(x, y-2 \xi) \beta(x, \xi)\right. \\
& -\int_{0}^{y-2 \xi} h^{n}(x, \tau) w_{t}^{n}(x, \xi, y-\xi-\tau) d \tau+\nu^{2} \exp (r(x, 0)(y-\xi) / 2) \\
& \times \frac{\partial}{\partial x} L_{0}\left[\varphi_{8}^{n}(x, y-\xi) \exp (-r(x, 0)(y-\xi) / 2),\left(r_{00 t}^{n}+\frac{\partial \varphi_{4}^{n}}{\partial x}\right)(x, \xi, y-\xi)\right] \\
& +\frac{r(x, 0)}{2} \frac{\partial}{\partial x} L_{0}\left[\varphi_{8}^{n}(x, y-\xi) \exp (-r(x, 0)(y-\xi) / 2),\left(r_{00}^{n}+\frac{\partial \varphi_{2}^{n}}{\partial x}\right)(x, \xi, y-\xi)\right] \\
& \left.+\frac{1}{2} \frac{\partial}{\partial x}\left[\varphi_{2}^{n}(x, y-2 \xi) \exp (-r(x, 0)(y-2 \xi) / 2)\left(r_{00}^{n}+\frac{\partial \varphi_{2}^{n}}{\partial x}\right)(x, \xi, \xi)\right]\right\} d \xi \\
& \varphi_{6}^{n+1}(x, y)=\varphi_{6}^{0}(x, y)+\int_{0}^{y}(y-\xi)\left(h^{n}(x, \xi)+r(x, 0) \varphi_{7}^{n}(x, \xi)-\frac{r^{2}(x, 0)}{4} \varphi_{6}^{n}(x, \xi)\right) d \xi, \\
& \varphi_{7}^{n+1}(x, y)=\varphi_{7}^{0}(x)+\int_{0}^{y}\left(h^{n}(x, \xi)+r(x, 0) \varphi_{7}^{n}(x, \xi)-\frac{r^{2}(x, 0)}{4} \varphi_{6}^{n}(x, \xi)\right) d \xi \\
& \varphi_{8}^{n+1}(x, y)=\varphi_{8}^{0}(x, y)+\int_{0}^{y}(y-\xi)\left(c_{0}(x) \varphi_{8}^{n}(x, \xi)-L\left[\varphi_{8}^{n}, h^{n}\right]\right)(x, \xi) d \xi,
\end{aligned}
$$




$$
\varphi_{9}^{n+1}(x, y)=\varphi_{9}^{0}(x)+\int_{0}^{y}\left(c_{0}(x) \varphi_{8}^{n}(x, \xi)-L\left[\varphi_{8}^{n}, h^{n}\right]\right)(x, \xi) d \xi
$$

где

$$
\begin{aligned}
& w^{n}(x, y, t)=\varphi_{1}^{n}(x, y, t)+\frac{1}{2}\left\{\frac{a}{2}\left(\varphi_{8}^{n}(x, t+y)+\varphi_{8}^{n}(x, t-y)\right)\right. \\
& \left.+\int_{0}^{t+y} \varphi_{8}^{n}(x, \tau) \tilde{g}_{0}(x, t+y-\tau) d \tau+\int_{0}^{t-y} \varphi_{8}^{n}(x, \tau) \tilde{g}_{0}(x, t-y-\tau) d \tau\right\}, \\
& w_{t}^{n}(x, y, t)=\varphi_{3}^{n}(x, y, t)+\frac{a}{4}\left[\varphi_{9}^{n}(x, t+y)+\varphi_{9}^{n}(x, t-y)\right]+\frac{1}{2} g_{0}(x, 0)\left[\varphi_{8}^{n}(x, t+y)\right. \\
& \left.+\varphi_{8}^{n}(x, t-y)\right]+\frac{1}{2}\left(\int_{0}^{t+y} \varphi_{8}^{n}(x, t-\tau) \tilde{g}_{0 t}(x, \tau) d \tau+\int_{0}^{t-y} \varphi_{8}^{n}(x, t-\tau) \tilde{g}_{0 t}(x, \tau) d \tau\right), \\
& h^{n}(x, y)=\frac{1}{2}\left[\varphi_{5}^{n}(x, y)+c_{0}(x) \varphi_{8}^{n}(x, y)\right]+\frac{1}{a}\left[g_{0}(x, 0) \varphi_{9}^{n}(x, y)+\varphi_{8}^{n}(x, y) \tilde{g}_{0 t}(x, 0)\right], \\
& \varphi_{2}^{n}(x, y, t)=\exp (k(x, 0) t / 2) L_{0}\left[\varphi_{6}^{n}, w^{n}\right] \text {, } \\
& \varphi_{4}^{n}(x, y, t)=\exp (k(x, 0) t / 2)\left(\frac{k(x, 0)}{2} L_{0}\left[\varphi_{6}^{n}, w^{n}\right]+L_{0}\left[\varphi_{6}^{n}, w_{t}^{n}\right]+\varphi_{6}^{n}(x, t-y) \beta(x, y)\right) \text {, } \\
& k_{00}^{n}(x, y, t)=\frac{\nu^{2} a}{2} \exp (k(x, 0)(t-y) / 2)\left[\left(k_{x x}(x, 0) \frac{y}{2}+k_{x}^{2}(x, 0) \frac{y^{2}}{4}+\frac{k_{x}^{2}(x, 0)(t-y)}{2}\right)\right. \\
& \left.\times \varphi_{8}^{n}(x, t-y)+k_{x}(x, 0) \frac{\partial \varphi_{8}^{n}}{\partial x}(x, t-y)\right], \\
& r_{00}^{n}(x, y, t)=\frac{\partial}{\partial x}\left(\varphi_{6}^{n}(x, t-y) \exp (k(x, 0) t / 2)\right), \\
& k_{00 t}^{n}(x, y, t)=\frac{k(x, 0)}{2} k_{00}^{n}(x, y, t)+\frac{\nu^{2} a}{2} \exp (k(x, 0)(t-y) / 2)\left[\left(k_{x x}(x, 0) \frac{y}{2}+k_{x}^{2}(x, 0) \frac{y^{2}}{4}\right.\right. \\
& \left.\left.+\frac{k_{x}^{2}(x, 0)(t-y)}{2}\right) \varphi_{9}^{n}(x, t-y)+k_{x}(x, 0) \frac{\partial \varphi_{9}^{n}}{\partial x}(x, t-y)+\frac{k_{x}^{2}(x, 0)}{2} \varphi_{8}^{n}(x, t-y)\right], \\
& r_{00 t}^{n}(x, y, t)=\frac{\partial}{\partial x}\left[\exp (k(x, 0) t / 2)\left(\varphi_{7}^{n}(x, t-y)+\frac{k(x, 0)}{2} \varphi_{6}^{n}(x, t-y)\right)\right] .
\end{aligned}
$$

Определим функцию $s^{\prime}(y)$ формулой

$$
s^{\prime}(y)=\frac{s+\gamma^{n}(y)}{2}, \quad \gamma^{n}(y)=s_{0}-\frac{y}{b_{n}} .
$$

Введем обозначение $\psi_{i}^{n}=\varphi_{i}^{n+1}-\varphi_{i}^{n}, i=1,3,5,6,7,8,9$. Тогда справедливы следующие соотношения:

$$
\begin{aligned}
\psi_{1}^{0}(x, y, t) & =\frac{1}{2} \int_{0}^{y} \int_{t-y+\xi}^{t+y-\xi}\left\{H(x, \xi) w^{0}(x, \xi, \tau)-\frac{a}{2} h^{0}(x, \tau-\xi)+k_{00}^{0}(x, \xi, \tau)\right. \\
& -\int_{0}^{\tau-\xi} h^{0}(x, \gamma) w^{0}(x, \xi, \tau-\gamma) d \gamma+\nu^{2} \exp (r(x, 0) \tau / 2)
\end{aligned}
$$




$$
\begin{aligned}
& \left.\times \frac{\partial}{\partial x} L_{0}\left[\varphi_{8}^{0}(x, \tau) \exp (-r(x, 0)(\tau) / 2),\left(r_{00}^{0}+\frac{\partial \varphi_{2}^{0}}{\partial x}\right)(x, \xi, \tau)\right]\right\} d \tau d \xi, \\
& \psi_{3}^{0}(x, y, t)=\frac{1}{2} \int_{-y}^{y}\left\{H(x, y-|\xi|) w^{0}(x, y-|\xi|, \xi+\tau)-\frac{a}{2} h^{0}(x, \xi+t-y+|\xi|)\right. \\
& +k_{00}^{0}(x, y-|\xi|, t+|\xi|)-\int_{0}^{t-y+\xi+|\xi|} h^{0}(x, \gamma) w^{0}(x, y-|\xi|, t+\xi-\gamma) d \gamma \\
& +\nu^{2} \exp (r(x, 0)(\xi+t) / 2) \frac{\partial}{\partial x} L_{0}\left[\varphi_{8}^{0}(x, \xi+t)\right. \\
& \left.\left.\times \exp (-r(x, 0)(\xi+t) / 2),\left(r_{00}+\frac{\partial \varphi_{2}^{0}}{\partial x}\right)(x, y-|\xi|, \xi+t)\right]\right\} \operatorname{sgn}(\xi) d \xi, \\
& \psi_{5}^{0}(x, y)=\int_{0}^{y}\left(\frac{2}{a} \tilde{g}_{0 t t}(x, y-\xi)-h^{0}(x, y-\xi)\right) \varphi_{8}^{0}(x, \xi) d \xi \\
& +\frac{1}{a} \int_{0}^{y / 2}\left\{H(x, \xi) w_{t}^{0}(x, \xi, y-\xi)+k_{00 t}^{0}(x, \xi, y-\xi)-\frac{1}{2} h^{0}(x, y-2 \xi) \beta(x, \xi)\right. \\
& -\int_{0}^{y-2 \xi} h^{0}(x, \tau) w_{t}^{0}(x, \xi, y-\xi-\tau) d \tau+\nu^{2} \exp (r(x, 0)(y-\xi) / 2) \\
& \times\left\{\frac{\partial}{\partial x} L_{0}\left[\varphi_{8}^{0}(x, y-\xi) \exp (-r(x, 0)(y-\xi) / 2),\left(r_{00 t}^{0}+\frac{\partial \varphi_{4}^{0}}{\partial x}\right)(x, \xi, y-\xi)\right]\right. \\
& +\frac{r(x, 0)}{2} \frac{\partial}{\partial x} L_{0}\left[\varphi_{8}^{0}(x, y-\xi) \exp (-r(x, 0)(y-\xi) / 2),\left(r_{00}^{0}+\frac{\partial \varphi_{2}^{0}}{\partial x}\right)(x, \xi, y-\xi)\right] \\
& \left.\left.+\frac{1}{2} \frac{\partial}{\partial x}\left[\varphi_{2}^{0}(x, y-2 \xi) \exp (-r(x, 0)(y-2 \xi) / 2)\left(r_{00}^{0}+\frac{\partial \varphi_{2}^{0}}{\partial x}(x, \xi, \xi)\right)\right]\right\}\right\} d \xi \\
& \psi_{6}^{0}(x, y)=\int_{0}^{y}(y-\xi)\left(h^{0}(x, \xi)+r(x, 0) \varphi_{7}^{0}(x, \xi)-\frac{r^{2}(x, 0)}{4} \varphi_{6}^{0}(x, \xi)\right) d \xi, \\
& \psi_{7}^{0}(x, y)=\int_{0}^{y}\left(h^{0}(x, \xi)+r(x, 0) \varphi_{7}^{0}(x, \xi)-\frac{r^{2}(x, 0)}{4} \varphi_{6}^{0}(x, \xi)\right) d \xi, \\
& \psi_{8}^{0}(x, y)=\int_{0}^{y}(y-\xi)\left(c_{0}(x) \varphi_{8}^{0}(x, \xi)-L\left[\varphi_{8}^{0}, h^{0}\right]\right) d \xi \\
& \psi_{9}^{0}(x, y)=\int_{0}^{y}\left(c_{0}(x) \varphi_{8}^{0}(x, \xi)-L\left[\varphi_{8}^{0}, h^{0}\right]\right) d \xi
\end{aligned}
$$




$$
\begin{aligned}
& \psi_{1}^{n+1}(x, y, t)=\frac{1}{2} \int_{0}^{y} \int_{t-y+\xi}^{t+y-\xi}\left\{H(x, \xi) \hat{w}^{n}(x, \xi, \tau)-\frac{a}{2} \hat{h}^{n}(x, \tau-\xi)+\hat{k}_{00}^{n}(x, \xi, \tau)\right. \\
& -\int_{0}^{\tau-\xi}\left(\hat{h}^{n}(x, \gamma) w^{n+1}(x, \xi, \tau-\gamma)-h^{n}(x, \gamma) \hat{w}^{n}(x, \xi, \tau-\gamma)\right) d \gamma \\
& +\nu^{2} \exp (r(x, 0) \tau / 2) \frac{\partial}{\partial x}\left\{L_{0}\left[\varphi_{8}^{n+1}(x, \tau) \exp (-r(x, 0)(\tau) / 2),\left(\hat{r}_{00}^{n}+\frac{\partial \psi_{2}^{n}}{\partial x}\right)(x, \xi, \tau)\right]\right. \\
& \left.\left.-\int_{0}^{\tau-\xi} \psi_{8}^{n}(x, \gamma) \exp (-r(x, 0) \gamma / 2)\left(r_{00}^{n}+\frac{\partial \varphi_{2}^{n}}{\partial x}\right)(x, \xi, \tau-\gamma) d \gamma\right\}\right\} d \tau d \xi \\
& \psi_{3}^{n+1}(x, y, t)=\frac{1}{2} \int_{-y}^{y}\left\{H(x, y-|\xi|) \hat{w}^{n}(x, y-|\xi|, \xi+\tau)-\frac{a}{2} \hat{h}^{n}(x, \xi+t-y+|\xi|)\right. \\
& +\hat{k}_{00}^{n}(x, y-|\xi|, t+|\xi|)-\int_{0}^{t-y+\xi+|\xi|}\left(\hat{h}^{n}(x, \gamma) w^{n+1}(x, y-|\xi|, t+\xi-\gamma)\right. \\
& \left.-h^{n}(x, \gamma) \hat{w}^{n}(x, y-|\xi|, t+\xi-\gamma)\right) d \gamma+\nu^{2} \exp (r(x, 0)(\xi+t) / 2) \\
& \times \frac{\partial}{\partial x}\left\{L_{0}\left[\varphi_{8}^{n+1}(x, \xi+t) \exp (-r(x, 0)(\xi+t) / 2),\left(\hat{r}_{00}^{n}+\frac{\partial \psi_{2}^{n}}{\partial x}\right)(x, y-|\xi|, \xi+t)\right]\right. \\
& \left.\left.-\int_{0}^{t-y+\xi+|\xi|} \psi_{8}^{n}(x, \gamma) \exp (-r(x, 0) \gamma / 2)\left(r_{00}^{n}+\frac{\partial \varphi_{2}^{n}}{\partial x}\right)(x, y-\xi, \xi+t-\gamma) d \gamma\right\}\right\} \operatorname{sgn}(\xi) d \xi, \\
& \left.\left.\psi_{5}^{n+1}(x, y)=\int_{0}^{y}\left(\frac{2}{a} \psi_{8}^{n}(x, \xi) \tilde{g}_{0 t t}(x, y-\xi)-\hat{h}^{n}(x, y-\xi)\right) \varphi_{8}^{n+1}(x, \xi)+h^{n}(x, y-\xi)\right) \psi_{8}^{n}(x, \xi)\right) d \xi \\
& +\frac{1}{a} \int_{0}^{y / 2}\left\{H(x, \xi) \hat{w}_{t}^{n}(x, \xi, y-\xi)+\hat{k}_{00 t}^{n}(x, \xi, y-\xi)-\frac{1}{2} \hat{h}^{n}(x, y-2 \xi) \beta(x, \xi)\right. \\
& -\int_{0}^{y-2 \xi}\left(\hat{h}^{n}(x, \tau) w_{t}^{n+1}(x, \xi, y-\xi-\tau)-h^{n}(x, \tau) \hat{w}_{t}^{n}(x, \xi, y-\xi-\tau)\right) d \tau \\
& +\nu^{2} \exp (r(x, 0)(y-\xi) / 2)\left\{\frac { \partial } { \partial x } \left\{L _ { 0 } \left[\varphi_{8}^{n+1}(x, y-\xi) \exp (-r(x, 0)(y-\xi) / 2),\left(\hat{r}_{00 t}^{n}+\frac{\partial \psi_{4}^{n}}{\partial x}\right)\right.\right.\right. \\
& \left.\times(x, \xi, y-\xi)]-\int_{0}^{y-2 \xi} \psi_{8}^{n}(x, \tau) \exp (-r(x, 0) \tau / 2)\left(r_{00 t}^{n}+\frac{\partial \varphi_{4}^{n}}{\partial x}\right)(x, \xi, y-\xi-\tau) d \tau\right\} \\
& +\frac{r(x, 0)}{2} \frac{\partial}{\partial x}\left\{L_{0}\left[\varphi_{8}^{n+1}(x, \tau) \exp (-r(x, 0)(y-\xi) / 2),\left(\hat{r}_{00}^{n}+\frac{\partial \psi_{2}^{n}}{\partial x}\right)(x, \xi, y-\xi)\right]\right. \\
& \left.-\int_{0}^{y-2 \xi} \psi_{8}^{n}(x, \gamma) \exp (-r(x, 0) \tau / 2)\left(r_{00}^{n}+\frac{\partial \varphi_{2}^{n}}{\partial x}\right)(x, \xi, y-\xi-\tau) d \gamma\right\} \\
& +\frac{1}{2} \frac{\partial}{\partial x}\left[\operatorname { e x p } ( - r ( x , 0 ) ( y - 2 \xi ) / 2 ) \left(\varphi_{8}^{n+1}(x, y-2 \xi)\left(\hat{r}_{00}^{n}+\frac{\partial \psi_{2}^{n}}{\partial x}\right)(x, \xi, \xi)\right.\right. \\
& \left.\left.\left.-\psi_{8}^{n}(x, y-2 \xi)\left(r_{00}^{n}+\frac{\partial \varphi_{2}^{n}}{\partial x}\right)(x, \xi, \xi)\right)\right]\right\} d \xi
\end{aligned}
$$




$$
\begin{aligned}
\psi_{6}^{n+1}(x, y) & =\int_{0}^{y}(y-\xi)\left(\hat{h}^{n}(x, \xi)+r(x, 0) \psi_{7}^{n}(x, \xi)-\frac{r^{2}(x, 0)}{4} \psi_{6}^{n}(x, \xi)\right) d \xi \\
\psi_{7}^{n+1}(x, y) & =\int_{0}^{y}\left(\hat{h}^{n}(x, \xi)+r(x, 0) \psi_{7}^{n}(x, \xi)-\frac{r^{2}(x, 0)}{4} \psi_{6}^{n}(x, \xi)\right) d \xi \\
\psi_{8}^{n+1}(x, y) & =\int_{0}^{y}(y-\xi)\left(c_{0}(x) \psi_{8}^{n}(x, \xi)-L\left[\varphi_{8}^{n}, \hat{h}^{n}\right]-\int_{0}^{\xi} \psi_{8}^{n}(x, \xi-\tau) h^{n+1}(x, \tau) d \tau\right) d \xi \\
\psi_{9}^{n+1}(x, y) & =\int_{0}^{y}\left(c_{0}(x) \psi_{8}^{n}(x, \xi)-L\left[\varphi_{8}^{n}, \hat{h}^{n}\right]-\int_{0}^{\xi} \psi_{8}^{n}(x, \xi-\tau) h^{n+1}(x, \tau) d \tau\right) d \xi
\end{aligned}
$$

где

$$
\begin{aligned}
\hat{k}_{00}^{n}(x, y, t) & =\frac{\nu^{2} a}{2} \exp (k(x, 0)(t-y) / 2)\left[\left(k_{x x}(x, 0) \frac{y}{2}+k_{x}^{2}(x, 0) \frac{y^{2}}{4}+\frac{k_{x}^{2}(x, 0)(t-y)}{2}\right)\right. \\
& \left.\times \psi_{8}^{n}(x, t-y)+k_{x}(x, 0) \frac{\partial \psi_{8}^{n}}{\partial x}(x, t-y)\right], \\
\hat{r}_{00}^{n}(x, y, t)= & \frac{\partial}{\partial x}\left(\psi_{6}^{n}(x, t-y) \exp (k(x, 0) t / 2)\right), \\
\hat{k}_{00 t}^{n}(x, y, t)= & \frac{k(x, 0)}{2} k_{00}^{n}(x, y, t)+\frac{\nu^{2} a}{2} \exp (k(x, 0)(t-y) / 2)\left[\left(k_{x x}(x, 0) \frac{y}{2}+k_{x}^{2}(x, 0) \frac{y^{2}}{4}\right.\right. \\
& \left.\left.+\frac{k_{x}^{2}(x, 0)(t-y)}{2}\right) \psi_{9}^{n}(x, t-y)+k_{x}(x, 0) \frac{\partial \psi_{9}^{n}}{\partial x}(x, t-y)+\frac{k_{x}^{2}(x, 0)}{2} \psi_{8}^{n}(x, t-y)\right], \\
\hat{r}_{00 t}^{n}(x, y, t)= & \frac{\partial}{\partial x}\left[\exp (k(x, 0) t / 2)\left(\psi_{7}^{n}(x, t-y)+\frac{k(x, 0)}{2} \psi_{6}^{n}(x, t-y)\right)\right], \\
\hat{w}^{n}(x, y, t)= & \psi_{1}^{n}(x, y, t)+\frac{1}{2}\left\{\frac{a}{2}\left(\psi_{8}^{n}(x, t+y)+\psi_{8}^{n}(x, t-y)\right)\right. \\
& \left.+\int_{0}^{t+y} \psi_{8}^{n}(x, \tau) \tilde{g}_{0}(x, t+y-\tau) d \tau+\int_{0}^{t-y} \psi_{8}^{n}(x, \tau) \tilde{g}_{0}(x, t-y-\tau) d \tau\right\}, \\
\psi_{2}^{n}(x, y, t)= & \exp (k(x, 0) t / 2)\left(L_{0}\left[\varphi_{6}^{n+1}, \hat{w}^{n}\right]-\int_{y}^{t} \psi_{6}^{n}(x, t-\tau) w^{n}(x, y, \tau) d \tau\right), \\
\hat{h}^{n}(x, y)= & \frac{1}{2}\left[\psi_{5}^{n}(x, y)+c_{0}(x) \psi_{8}^{n}(x, y)\right]+\frac{1}{a}\left[g_{0}(x, 0) \psi_{9}^{n}(x, y)+\psi_{8}^{n}(x, y) \tilde{g}_{0 t}(x, 0)\right], \\
\hat{w}_{t}^{n}(x, y, t)= & \psi_{3}^{n}(x, y, t)+\frac{a}{4}\left[\psi_{9}^{n}(x, t+y)+\psi_{9}^{n}(x, t-y)\right]+\frac{1}{2} g_{0}(x, 0)\left[\psi_{8}^{n}(x, t+y)+\psi_{8}^{n}(x, t-y)\right] \\
& \left.\frac{1}{2}(x, t-\tau) \tilde{g}_{0 t}(x, \tau) d \tau+\int_{8}^{n}(x, t-\tau) \tilde{g}_{0 t}(x, \tau) d \tau\right),
\end{aligned}
$$




$$
\begin{aligned}
& \psi_{4}^{n}(x, y, t)=\frac{k(x, 0)}{2} \psi_{2}^{n}(x, y, t)+\exp (k(x, 0) t / 2)\left(L_{0}\left[\varphi_{6}^{n+1}, \hat{w}_{t}^{n}\right]\right. \\
& \left.-\int_{y}^{t} \psi_{6}^{n}(x, y-\tau) w^{n}(x, y, \tau) d \tau-\psi_{6}^{n}(x, t-y) \beta(x, y)\right) .
\end{aligned}
$$

Покажем, что можно выбрать $b_{0} \in(0, T / 2)$ так, что для любого $n=0,1,2, \ldots$ будут выполнены неравенства

$$
\begin{gathered}
\lambda_{n}=\max \left\{\sup _{(y, t, s) \in F_{n}}\left[\left\|\psi_{1}^{n}\right\|_{s}(y, t) \frac{\left.\gamma^{n}(y)-s\right]}{y}\right], \sup _{(y, t, s) \in F_{n}}\left[\left\|\psi_{i}^{n}\right\|_{s}(y) \frac{\gamma^{n}(y)-s}{y}\right], i=6,8,\right. \\
\sup _{(y, t, s) \in F_{n}}\left[\left\|\psi_{3}^{n}\right\|_{s}(y, t) \frac{\left(\gamma^{n}(y)-s\right)^{2}}{y}\right], \sup _{(y, t, s) \in F_{n}}\left[\left\|\psi_{5}^{n}\right\|_{s}(y) \frac{\left(\gamma^{n}(y)-s\right)^{3}}{y}\right], \\
\left.\sup _{(y, t, s) \in F_{n}}\left[\left\|\psi_{i}^{n}\right\|_{s}(y) \frac{\left(\gamma^{n}(y)-s\right)^{2}}{y}\right], i=7,9\right\}<\infty \\
\left\|\varphi_{1}^{n+1}-\varphi_{1}^{0}\right\|_{s}(y, t) \leqslant R, \quad\left\|\varphi_{i}^{n+1}-\varphi_{i}^{0}\right\|_{s}(y) \leqslant R, \quad i=6,8 \\
\left\|\varphi_{3}^{n+1}-\varphi_{3}^{0}\right\|_{s}(y, t) \leqslant \frac{R}{s_{0}-s}, \quad\left\|\varphi_{i}^{n+1}-\varphi_{i}^{0}\right\|_{s}(y) \leqslant \frac{R}{s_{0}-s}, \quad i=7,9, \\
\left\|\varphi_{5}^{n+1}-\varphi_{5}^{0}\right\|_{s}(y) \leqslant \frac{R}{\left(s_{0}-s\right)^{2}}, \quad(y, t, s) \in F_{n+1},
\end{gathered}
$$

где

$$
F_{n}=\left\{(y, t, s):(y, t) \in G_{T}, 0 \leqslant y \leqslant b_{n}\left(s_{0}-s\right), 0<s<s_{0}\right\} .
$$

Действительно, используя соотношения для $\psi_{i}^{n}$ при $n=0$, получим:

$$
\begin{gathered}
\left\|\psi_{1}^{0}\right\|_{s}(y) \leqslant \int_{0}^{y}(y-\xi)\left\{R\left\|w^{0}\right\|_{s}+\frac{a}{2}\left\|h^{0}\right\|_{s}+\left\|k_{00}^{0}\right\|_{s}+T\left\|h^{0}\right\|_{s}\left\|w^{0}\right\|_{s}\right. \\
\left.+R\left\|\frac{\partial}{\partial x} L_{0}\left[\varphi_{8}^{0}(x, \tau) \exp (-r(x, 0) \tau / 2),\left(r_{00}^{0}+\frac{\partial \varphi_{2}^{0}}{\partial x}\right)\right]\right\|_{s}(\xi, \tau)\right\} d \xi,
\end{gathered}
$$

где

$$
\begin{gathered}
\left\|w^{0}\right\|_{s} \leqslant R\left(1+\frac{a}{2}+2 T R\right), \quad\left\|h^{0}\right\|_{s} \leqslant R\left(\frac{1}{2}+R\left(1+\frac{4}{a}\right)\right), \\
\left\|\varphi_{2}^{0}\right\|_{s}(y, t) \leqslant R(1+R T)\left\|w^{0}\right\|_{s} \leqslant R(1+R T) R\left(\frac{1}{2}+R\left(1+\frac{4}{a}\right)\right), \\
\left\|k_{00}^{0}\right\|_{s}(y, t) \leqslant \frac{a}{2} R^{2}\left(R\left(1+\frac{T}{2}\right)+\left\|\frac{\partial \varphi_{8}^{0}}{\partial x}\right\|_{s}\right), \quad s \in\left(0, s_{0}\right), \quad(y, t) \in G_{T} .
\end{gathered}
$$

Воспользуемся неравенством (2.35) для оценки $\left\|\frac{\partial \varphi_{8}^{0}}{\partial x}\right\|_{s},\left\|r_{00}^{0}\right\|_{s},\left\|\frac{\partial \varphi_{2}^{0}}{\partial x}\right\|_{s},\left\|\frac{\partial}{\partial x} L_{0}\right\|_{s}$ :

$$
\begin{gathered}
\left\|\frac{\partial \varphi_{8}^{0}}{\partial x}\right\|_{s}(y) \leqslant \frac{R}{s^{\prime}(y)-s}, \quad\left\|r_{00}^{0}\right\|_{s}(y, t) \leqslant \frac{R^{2}}{s^{\prime}(y)-s}, \\
\left\|\frac{\partial}{\partial x} L_{0}\left[\varphi_{8}^{0} \exp (-r(x, 0)(t \pm y \mp \xi) / 2), r_{00}^{0}+\frac{\partial \varphi_{2}^{0}}{\partial x}\right]\right\|_{s}(y, t) \\
\leqslant \frac{1}{s^{\prime}(y)-s}\left(\left\|r_{00}^{0}\right\|_{s^{\prime}}+\frac{\left\|\varphi_{2}^{0}\right\|_{s^{\prime}}}{s^{\prime}(y)-s}+2 T R^{2}\left(\left\|r_{00}^{0}\right\|_{s^{\prime}}+\frac{\left\|\varphi_{2}^{0}\right\|_{s^{\prime}}}{s^{\prime}(y)-s}\right)\right) \leqslant \frac{R^{2}+\left\|\varphi_{2}^{0}\right\|_{s^{\prime}}\left(1+2 T R^{2}\right) .}{\left(s^{\prime}(y)-s\right)^{2}} .
\end{gathered}
$$


Взяв функцию $s^{\prime}(\xi)$ из (3.1) для $n=0$, с учетом вышеприведенных неравенств, имеем:

$$
\left\|\psi_{1}^{0}\right\|_{s}(y, t) \leqslant \mu_{0}\left(a, T, R, s_{0}\right) \int_{0}^{y} \frac{y-\xi}{\left(\gamma^{0}(y)-s\right)^{2}} d \xi \leqslant b_{0} \mu_{0}\left(a, T, R, s_{0}\right) \frac{y}{\gamma^{0}(y)-s} .
$$

Далее,

$$
\begin{gathered}
\left\|\psi_{3}^{0}\right\|_{s}(y, t) \leqslant \frac{1}{2} \int_{-y}^{y}\left\{R\left\|w^{0}\right\|_{s}+\frac{a}{2}\left\|h^{0}\right\|_{s}+\left\|k_{00}^{0}\right\|_{s}+3 T\left\|h^{0}\right\|_{s}\left\|w^{0}\right\|_{s}\right. \\
\left.+R\left\|\frac{\partial}{\partial x} L_{0}\left[\varphi_{8}^{0}(x, \xi+t) \exp (-r(x, 0)(\xi+t) / 2),\left(r_{00}^{0}+\frac{\partial \varphi_{2}^{0}}{\partial x}\right)\right]\right\|_{s}(y-|\xi|, \xi+t)\right\} d \xi \\
\leqslant \frac{1}{2} \int_{-y}^{y} \frac{\mu_{1}\left(a, T, R, s_{0}\right)}{\left(\gamma^{0}(y)-s\right)^{2}} d \xi \leqslant \mu_{1}\left(a, T, R, s_{0}\right) \frac{y}{\left(\gamma^{0}(y)-s\right)^{2}}, \\
\left\|\psi_{5}^{0}\right\|_{s}(y) \leqslant \frac{a}{2} \int_{0}^{y} R\left(\frac{2}{a} R+\left\|h^{0}\right\|_{s}\right) d \xi+\frac{1}{a} \int_{0}^{y / 2}\left\{R\left\|w_{t}^{0}\right\|_{s}+\frac{1}{2} R\left\|h^{0}\right\|_{s}+\left\|k_{00 t}^{0}\right\|_{s}\right. \\
+T\left\|h^{0}\right\|_{s}\left\|w_{t}^{0}\right\|_{s}+R\left\{\left\|\frac{\partial}{\partial x} L_{0}\left[\varphi_{8}^{0}(x, y-\xi) \exp (-r(x, 0)(y-\xi) / 2), r_{00 t}^{0}+\frac{\partial \varphi_{4}^{0}}{\partial x}\right]\right\|_{s}\right. \\
+R\left\|\frac{\partial}{\partial x} L_{0}\left[\varphi_{8}^{0}(x, y-\xi) \exp (-r(x, 0)(y-\xi) / 2), r_{00}^{0}+\frac{\partial \varphi_{2}^{0}}{\partial x}\right]\right\|_{s} \\
\left.\left.+\frac{1}{2}\left\|\frac{\partial}{\partial x}\left(\varphi_{8}^{0}(x, y-\xi) \exp (-r(x, 0)(y-\xi) / 2)\left(r_{00}^{0}+\frac{\partial \varphi_{2}^{0}}{\partial x}\right)\right)\right\|_{s}\right\}\right\} d \xi
\end{gathered}
$$

где

$$
\begin{gathered}
\left\|w_{t}^{0}\right\|_{s} \leqslant R\left(1+\frac{a}{2}+R(1+T)\right), \quad\left\|\varphi_{4}^{0}\right\|_{s} \leqslant R(1+T R)\left(R\left\|w^{0}\right\|_{s}+\left\|w_{t}^{0}\right\|_{s}\right)+R^{3}, \\
\left\|k_{00 t}^{0}\right\|_{s}(y, t) \leqslant R\left\|k_{00 t}^{0}\right\|_{s}+\frac{a}{2} R^{2}\left(R(1+T R)+R^{2}+\left\|\frac{\partial \varphi_{9}^{0}}{\partial x}\right\|_{s}(y)\right), \\
\left\|r_{00 t}^{0}\right\|_{s}(y, t) \leqslant \frac{R^{2}(1+R)}{s^{\prime}(y)-s}, \quad\left\|\frac{\partial \varphi_{9}^{0}}{\partial x}\right\|_{s}(y) \leqslant \frac{R}{s^{\prime}(y)-s}, \quad s \in\left(0, s_{0}\right), \quad(y, t) \in G_{T} .
\end{gathered}
$$

Из вышеприведенных оценок следует, что

$$
\left\|\psi_{5}^{0}\right\|_{s}(y) \leqslant \mu_{2}\left(a, T, R, s_{0}\right) \frac{y}{\left(\gamma^{0}(y)-s\right)^{2}} \leqslant s_{0} \mu_{2}\left(a, T, R, s_{0}\right) \frac{y}{\left(\gamma^{0}(y)-s\right)^{3}} .
$$

Поступая аналогично, имеем

$$
\begin{aligned}
& \left\|\psi_{6}^{0}\right\|_{s}(y) \leqslant \int_{0}^{y}(y-\xi)\left(\left\|h^{0}\right\|_{s}+2 R^{2}\right) d \xi \leqslant s_{0} R T\left(1 / 2+2 R+R\left(1+\frac{4}{a}\right)\right) \frac{y}{\gamma^{0}(y)-s}, \\
& \left\|\psi_{7}^{0}\right\|_{s}(y) \leqslant \int_{0}^{y}\left(\left\|h^{0}\right\|_{s}+2 R^{2}\right) d \xi \leqslant s_{0}^{2} R\left(1 / 2+2 R+R\left(1+\frac{4}{a}\right)\right) \frac{y}{\left(\gamma^{0}(y)-s\right)^{2}},
\end{aligned}
$$




$$
\begin{aligned}
\left\|\psi_{8}^{0}\right\|_{s}(y) & \leqslant \int_{0}^{y}(y-\xi)\left(R^{2}+\left\|h^{0}\right\|_{s}(1+T R)\right) d \xi \\
& \leqslant s_{0} R T\left(R+\left(1 / 2+R\left(1+\frac{4}{a}\right)\right)(1+T R)\right) \frac{y}{\gamma^{0}(y)-s} \\
\left\|\psi_{9}^{0}\right\|_{s}(y) & \leqslant \int_{0}^{y}\left(R^{2}+R\left(1 / 2+R\left(1+\frac{4}{a}\right)\right)(1+T R)\right) d \xi \\
& \leqslant s_{0}^{2} R\left(R+\left(1 / 2+R\left(1+\frac{4}{a}\right)\right)(1+T R)\right) \frac{y}{\gamma^{0}(y)-s} .
\end{aligned}
$$

Из этих оценок следует выполнение неравенств (3.2) при $n=0$. Кроме того, для $(y, t, s) \in F_{1}$ находим

$$
\begin{aligned}
& \left\|\varphi_{1}^{1}-\varphi_{1}^{0}\right\|_{s}(y, t)=\left\|\psi_{1}^{0}\right\|_{s}(y, t) \leqslant \frac{\lambda_{0} y}{\gamma^{0}(y)-s} \leqslant \frac{\lambda_{0} b_{1}}{1-b_{1} / b_{0}}=\lambda_{0} b_{0}, \\
& \left\|\varphi_{3}^{1}-\varphi_{3}^{0}\right\|_{s}(y, t)=\left\|\psi_{3}^{0}\right\|_{s}(y, t) \leqslant \frac{\lambda_{0} y}{\left(\gamma^{0}(y)-s\right)^{2}} \leqslant \frac{2 \lambda_{0} b_{0}}{s_{0}-s} \\
& \left\|\varphi_{5}^{1}-\varphi_{5}^{0}\right\|_{s}(y)=\left\|\psi_{5}^{0}\right\|_{s}(y, t) \leqslant \frac{\lambda_{0} y}{\left(\gamma^{0}(y)-s\right)^{3}} \leqslant \frac{4 \lambda_{0} b_{0}}{\left(s_{0}-s\right)^{3}}, \\
& \left\|\varphi_{6}^{1}-\varphi_{6}^{0}\right\|_{s}(y)=\left\|\psi_{6}^{0}\right\|_{s}(y, t) \leqslant \frac{\lambda_{0} y}{\gamma^{0}(y)-s} \leqslant \frac{\lambda_{0} b_{1}}{1-b_{1} / b_{0}}=\lambda_{0} b_{0}, \\
& \left\|\varphi_{7}^{1}-\varphi_{7}^{0}\right\|_{s}(y)=\left\|\psi_{7}^{0}\right\|_{s}(y, t) \leqslant \frac{\lambda_{0} y}{\left(\gamma^{0}(y)-s\right)^{2}} \leqslant \frac{2 \lambda_{0} b_{0}}{s_{0}-s} \\
& \left\|\varphi_{8}^{1}-\varphi_{8}^{0}\right\|_{s}(y)=\left\|\psi_{8}^{0}\right\|_{s}(y, t) \leqslant \frac{\lambda_{0} y}{\gamma^{0}(y)-s} \leqslant \frac{\lambda_{0} b_{1}}{1-b_{1} / b_{0}}=\lambda_{0} b_{0}, \\
& \left\|\varphi_{9}^{1}-\varphi_{9}^{0}\right\|_{s}(y)=\left\|\psi_{9}^{0}\right\|_{s}(y, t) \leqslant \frac{\lambda_{0} y}{\left(\gamma^{0}(y)-s\right)^{2}} \leqslant \frac{2 \lambda_{0} b_{0}}{s_{0}-s} .
\end{aligned}
$$

Если выбрать $b_{0}$ так, чтобы $4 \lambda_{0} b_{0} \leqslant R$, то неравенства (3.3) будут выполняться для $n=0$. Покажем методом индукции, что неравенства (3.2), (3.3) имеют место и для других $n$, если $b_{0}$ выбрать подходящим образом. Пусть неравенства (3.2), (3.3) справедливы для $n=0,1,2, \ldots, j$. Тогда для $(y, t, s) \in F_{j+1}$

$$
\begin{gathered}
\left\|\psi_{1}^{j+1}\right\|_{s}(y, t) \leqslant \int_{0}^{y}(y-\xi)\left\{R\left\|\hat{w}^{j}\right\|_{s}+\frac{a}{2}\left\|\hat{h}^{j}\right\|_{s}+\left\|\hat{k}_{00}^{j}\right\|_{s}\right. \\
+T\left(\left\|\hat{h}^{j}\right\|_{s}\left\|w^{j+1}\right\|_{s}+\left\|h^{j}\right\|_{s}\left\|\hat{w}^{j}\right\|_{s}\right)+R\left\{\left\|\frac{\partial}{\partial x} L_{0}\left[\varphi_{8}^{j+1} \exp (-r(x, 0) t / 2), \hat{r}_{00}^{j}+\frac{\partial \psi_{2}^{j}}{\partial x}\right]\right\|_{s}\right. \\
\left.\left.+T\left\|\frac{\partial}{\partial x}\left[\psi_{8}^{j} \exp (-r(x, 0) t / 2)\left(r_{00}^{j}+\frac{\partial \varphi_{2}^{j}}{\partial x}\right)\right]\right\|_{s}\right\}\right\} d \xi .
\end{gathered}
$$

Нетрудно видеть, что

$$
\left\|\hat{w}^{j}\right\|_{s}(y, t) \leqslant\left\|\psi_{1}^{j}\right\|_{s}+\frac{1}{2}(a+T R)\left\|\psi_{8}^{j}\right\|_{s} \leqslant(1+(a+T R) / 2) \frac{\lambda_{j} y}{\gamma^{j}(y)-s},
$$




$$
\begin{aligned}
\left\|\hat{h}^{j}\right\|_{s}(y) & \leqslant \frac{1}{2}\left\|\psi_{5}^{j}\right\|_{s}+R\left\|\psi_{8}^{j}\right\|_{s}+\frac{2}{a} R\left(\left\|\psi_{9}^{j}\right\|_{s}+\left\|\psi_{8}^{j}\right\|_{s}\right) \\
\leqslant & \left(1 / 2+R(1+2 / a) s_{0}+2 R s_{0}\right) \frac{\lambda_{j} y}{\left(\gamma^{j}(y)-s\right)^{3}}, \\
\left\|\hat{k}_{00}^{j}\right\|_{s}(y, t) & \leqslant \frac{a}{2} R^{2}\left((1+T R / 2)\left\|\psi_{8}^{j}\right\|_{s}+\frac{\left\|\psi_{8}^{j}\right\|_{s}}{s^{\prime}(y)-s}\right) \leqslant \frac{a}{2} R^{2}(3+T R / 2) \frac{\lambda_{j} y}{\left(\gamma^{j}(y)-s\right)^{2}}, \\
\left\|\hat{r}_{00}^{j}\right\|_{s}(y, t) & \leqslant R \frac{\lambda_{j} y}{\left(s^{\prime}(y)-s\right)\left(\gamma^{j}(y)-s\right)}, \\
\left\|\psi_{2}^{j}\right\|_{s}(y, t) & \leqslant R\left(\left\|\hat{w}^{j}\right\|_{s}(1+2 R T)+T\left\|\psi_{6}^{j}\right\|_{s}\left\|w^{j}\right\|_{s}\right) \\
& \leqslant R((1+2 R T)(1+a / 2+T R / 2)+T R(2+a+2 T R)) \frac{\lambda_{j} y}{\gamma^{j}(y)-s}, \\
\left\|\frac{\partial}{\partial x} L_{0}\left[\varphi_{8}^{j+1} \exp (-r(x, 0) t / 2), \hat{r}_{00}^{0}+\frac{\partial \psi_{2}^{j}}{\partial x}\right]\right\|_{s}(y, t) \leqslant \frac{1}{s^{\prime}(y)-s}(1+T R)\left(\left\|\hat{r}_{00}^{j}\right\|_{s}+\frac{\left\|\psi_{2}^{j}\right\|_{s}}{s^{\prime}(y)-s}\right) & \leqslant \mu_{3}(a, T, R) \frac{\lambda_{j} y}{\left(\gamma^{j}(y)-s\right)^{3}},
\end{aligned}
$$

где

$$
\left\|w^{j}\right\|_{s}(y, t) \leqslant\left\|\varphi_{1}^{j}\right\|_{s}+\frac{a}{2}\left\|\varphi_{8}^{j}\right\|_{s}+T R\left\|\varphi_{8}^{j}\right\|_{s} \leqslant R(2+a+2 T R) .
$$

Здесь и в дальнейших промежуточных выкладках функция $s^{\prime}$ определена равенством (3.1) при $n=j$ и использованы неравенства

$$
\begin{gathered}
\left\|\varphi_{i}^{j}\right\|_{s} \leqslant 2 R, \quad i=1,6,8 ; \quad\left\|\varphi_{5}^{j}\right\|_{s} \leqslant R \frac{1+s_{0}^{2}}{\left(s_{0}-s\right)^{2}} \\
\left\|\varphi_{i}^{j}\right\|_{s} \leqslant R \frac{1+s_{0}}{s_{0}-s}, \quad i=3,7,9
\end{gathered}
$$

справедливые согласно индуктивному предположению, а также очевидные неравенства $b_{j} \leqslant b_{0}, \gamma^{j+1}(y) \leqslant \gamma^{j}(y),\left\|\varphi_{2}^{j}\right\|_{s}(y, t) \leqslant R(1+2 R T)\left\|w^{j}\right\|_{s} \leqslant R^{2}(1+2 R T)(2+a+2 T R)$,

$$
\begin{gathered}
\left\|\varphi_{4}^{j}\right\|_{s} \leqslant R\left(R\left\|w^{j}\right\|_{s}(1+2 T R)+\left\|w_{t}\right\|_{s}(1+2 R T)+2 R^{2}\right) \leqslant \frac{\mu_{4}\left(a, T, R, s_{0}\right)}{s_{0}-s} \\
\left\|w_{t}^{j}\right\|_{s} \leqslant \frac{R\left(1+s_{0}\right)(1+a R / 2)+2 R^{2} s_{0}(1+T)}{s_{0}-s}
\end{gathered}
$$

Окончательно для $\psi_{1}^{j+1}$ получаем

$$
\left\|\psi_{1}^{j+1}\right\|_{s}(y, t) \leqslant \int_{0}^{y}(y-\xi) \mu_{5}\left(a, T, R, s_{0}\right) \frac{\lambda_{j} \xi}{\left(\gamma^{j}(\xi)-s\right)^{3}} d \xi \leqslant \lambda_{j} b_{0}^{2} \mu_{5}\left(a, T, R, s_{0}\right) \frac{y}{\gamma^{j+1}(y)-s} .
$$

Аналогичные рассуждения для $\psi_{i}^{j+1}, i=3,5,6,7,8,9$, приводят к неравенствам

$$
\left\|\psi_{3}^{j+1}\right\|_{s}(y, t) \leqslant \frac{1}{2} \int_{-y}^{y} \mu_{6}\left(a, T, R, s_{0}\right) \frac{\lambda_{j} \xi}{\left(\gamma^{j}(\xi)-s\right)^{3}} d \xi \leqslant \lambda_{j} b_{0} \mu_{6}\left(a, T, R, s_{0}\right) \frac{y}{\left(\gamma^{j+1}(y)-s\right)^{2}}
$$




$$
\begin{gathered}
\left\|\psi_{5}^{j+1}\right\|_{s}(y, t) \leqslant \int_{0}^{y}\left[\left(\frac{a}{2} R+\left\|h^{j}\right\|_{s}\right)\left\|\psi_{8}^{j}\right\|_{s}+\left\|\hat{h}^{j}\right\|_{s}\left\|\varphi_{8}^{j+1}\right\|_{s}\right] d \xi \\
+\frac{1}{a} \int_{0}^{y / 2}\left\{\left(R+y\left\|h^{j}\right\|_{s}\right)\left\|\hat{w}_{t}^{j}\right\|_{s}+\left(R / 2+y\left\|w_{t}^{j+1}\right\|_{s}\right)\left\|\hat{h}^{j}\right\|_{s}+\left\|\hat{k}_{00 t}^{j}\right\|_{s}\right. \\
+R \frac{\left.\| L_{0} \varphi_{8}^{j+1} \exp (-r(x, 0)(y-\xi) / 2), \hat{r}_{00 t}^{j}+\frac{\partial \psi_{4}^{j}}{\partial x}\right] \|_{s}}{s^{\prime}(\xi)-s} \\
+\frac{y R^{2}\left\|\psi_{8}^{j}\right\|_{s}\left(\left\|r_{00 t}^{j}\right\|_{s}+\left\|\frac{\partial \varphi_{4}^{j}}{\partial x}\right\|_{s}\right)}{s^{\prime}(\xi)-s}+R \frac{\left.\| L_{0} \varphi_{8}^{j+1} \exp (-r(x, 0)(y-\xi) / 2), \hat{r}_{00}^{j}+\frac{\partial \psi_{2}^{j}}{\partial x}\right] \|_{s}}{s^{\prime}(\xi)-s} \\
+\frac{y R^{2}\left\|\psi_{8}^{j}\right\|_{s}\left(\left\|r_{00}^{j}\right\|_{s}+\left\|\frac{\partial \varphi_{2}^{j}}{\partial x}\right\|_{s}\right)}{s^{\prime}(\xi)-s}+\frac{R^{2}}{2} \frac{\left\|\varphi_{8}^{j+1}\right\|\left\|_{s}\left(\left\|\hat{r}_{00}^{j}\right\|_{s}+\left\|\frac{\partial \psi_{2}^{j}}{\partial x}\right\|_{s}\right)+\right\| \psi_{8}^{j} \|_{s}\left(\left\|r_{00}^{j}\right\|_{s}+\left\|\frac{\partial \varphi_{2}^{j}}{\partial x}\right\|_{s}\right)}{s^{\prime}(\xi)-s} d \xi \\
\leqslant \int_{0}^{y} \mu_{7}\left(a, T, R, s_{0}\right) \frac{\lambda_{j} \xi}{\left(\gamma^{j}(\xi)-s\right)^{4}} d \xi \leqslant \lambda_{j} b_{0} \mu_{7}\left(a, T, R, s_{0}\right) \frac{y}{\left(\gamma^{j+1}(y)-s\right)^{3}},
\end{gathered}
$$

где

$$
\begin{aligned}
\left\|\hat{w}_{t}^{j}\right\|_{s}(y, t) & \leqslant\left\|\psi_{3}^{j}\right\|_{s}+\frac{a}{2}\left\|\psi_{9}^{j}\right\|_{s}+R(1+T)\left\|\psi_{8}^{j}\right\|_{s} \leqslant\left(1+\frac{a}{2}+s_{0} R(1+T)\right) \frac{\lambda_{j} y}{\left(\gamma^{j}(y)-s\right)^{2}}, \\
\left\|\hat{k}_{00 t}^{j}\right\|_{s}(y, t) & \leqslant R\left\|\hat{k}_{00}^{j}\right\|_{s}+\frac{a}{2} R^{2}\left[\left(1+T R / 2+\frac{1}{s^{\prime}(y)-s}\right)\left\|\psi_{9}^{j}\right\|_{s}+\frac{R}{2}\left\|\psi_{8}^{j}\right\|_{s}\right] \\
& \leqslant \frac{a}{2} R^{2}\left(s_{0} R(3+T R / 2)+s_{0}(1+T R / 2)+2 R+s_{0}^{2} R^{2}\right) \frac{\lambda_{j} y}{\left(\gamma^{j}(y)-s\right)^{3}},
\end{aligned}
$$

$\left\|\hat{r}_{00 t}^{j}\right\|_{s}(y, t) \leqslant 2 R\left(1+s_{0} R\right) \frac{\lambda_{j} y}{\left(\gamma^{j}(y)-s\right)^{3}}, \quad\left\|r_{00 t}^{j}\right\|_{s}(y, t) \leqslant \frac{2 R^{2}\left(1+s_{0}+2 R s_{0}\right)}{\left(s_{0}-s\right)\left(\gamma^{j}(y)-s\right)}$, $\left\|\psi_{4}^{j}\right\|_{s}(y, t) \leqslant R\left\|\psi_{2}^{j}\right\|_{s}(y, t)+R\left[\left(\left\|\hat{w}^{j}\right\|_{s}+\left\|\hat{w}_{t}^{j}\right\|_{s}\right)(1+2 T R)+\left\|\psi_{6}^{j}\right\|_{s}\left(\left(\left\|w^{j}\right\|_{s}+\left\|w_{t}^{j}\right\|_{s}\right) T+R\right)\right]$.

Далее

$$
\begin{aligned}
\left\|\psi_{6}^{j+1}\right\|_{s}(y) & \leqslant \int_{0}^{y}(y-\xi)\left(\frac{\lambda_{j} \xi\left(1 / 2+R(1+2 / a) s_{0}+2 R s_{0}\right)}{\left(\gamma^{j}(y)-s\right)^{3}}+\frac{\lambda_{j} \xi R}{\left(\gamma^{j}(\xi)-s\right)^{2}}+\frac{\lambda_{j} \xi R}{\gamma^{j}(\xi)-s}\right) d \xi \\
& \leqslant \lambda_{j} \mu_{8}\left(b_{0}, a, R, s_{0}\right) \frac{y}{\left(\gamma^{j+1}(y)-s\right)}, \\
\left\|\psi_{7}^{j+1}\right\|_{s}(y) & \leqslant \int_{0}^{y}\left(\frac{\lambda_{j} \xi\left(1 / 2+R(1+2 / a) s_{0}+2 R s_{0}\right)}{\left(\gamma^{j}(y)-s\right)^{3}}+\frac{\lambda_{j} \xi R}{\left(\gamma^{j}(\xi)-s\right)^{2}}+\frac{\lambda_{j} \xi R}{\gamma^{j}(\xi)-s}\right) d \xi \\
& \leqslant \lambda_{j} b_{0}^{-1} \mu_{8}\left(b_{0}, a, R, s_{0}\right) \frac{y}{\left(\gamma^{j+1}(y)-s\right)^{2}}, \\
\left\|\psi_{8}^{j+1}\right\|_{s}(y) & \leqslant \lambda_{j} \mu_{9}\left(b_{0}, a, R, s_{0}, T\right) \frac{y}{\gamma^{j+1}(y)-s}, \\
\left\|\psi_{9}^{j+1}\right\|_{s}(y) & \leqslant \lambda_{j} b_{0}^{-1} \mu_{9}\left(b_{0}, s_{0}, R, T\right) \frac{y}{\left(\gamma^{j+1}(y)-s\right)^{2}}, \quad(y, t, s) \in F_{j+1} .
\end{aligned}
$$

Из полученных оценок следует

$$
\lambda_{j+1} \leqslant \lambda_{j} \rho, \quad \lambda_{j+1} \leqslant \infty, \quad \rho=\max \left(\mu_{5}, \mu_{6}, \mu_{7}, \max \left(1, b_{0}^{-1}\right) \mu_{8}, \max \left(1, b_{0}^{-1}\right) \mu_{9}\right) .
$$


Вместе с тем, для $(y, t, s) \in F_{j+2}$

$$
\begin{aligned}
&\left\|\varphi_{i}^{j+2}-\varphi_{i}^{0}\right\|_{s} \leqslant \sum_{n=0}^{j+1}\left\|\psi_{i}^{n}\right\|_{s} \leqslant \sum_{n=0}^{j+1} \frac{\lambda_{n} y}{\gamma^{n}(y)-s} \\
& \leqslant \sum_{n=0}^{j+1} \frac{\lambda_{n} b_{j+2}}{1-b_{i+2} / b_{n}} \leqslant \sum_{n=0}^{j+1} \lambda_{n} b_{n}(n+1)^{2} \leqslant \lambda_{0} b_{0} \sum_{n=0}^{j+1} \rho^{n}(n+1)^{2}, \quad i=1,6,8, \\
&\left\|\varphi_{5}^{j+2}-\varphi_{5}^{0}\right\|_{s}(y) \leqslant \sum_{n=0}^{j+1}\left\|\psi_{5}^{n}\right\|_{s}(y) \leqslant \sum_{n=0}^{j+1} \frac{\lambda_{n} y}{\left(\gamma^{n}(y)-s\right)^{3}} \\
& \leqslant \frac{1}{\left(s_{0}-s\right)^{2}} \sum_{n=0}^{j+1} \frac{\lambda_{n} b_{j+2}}{\left(1-b_{i+2} / b_{n}\right)^{3}} \leqslant \frac{\lambda_{0} b_{0}}{\left(s_{0}-s\right)^{2}} \sum_{n=0}^{j+1} \rho^{n}(n+1)^{6}, \\
&\left\|\varphi_{i}^{j+2}-\varphi_{i}^{0}\right\|_{s} \leqslant \sum_{n=0}^{j+1}\left\|\psi_{i}^{n}\right\|_{s} \leqslant \sum_{n=0}^{j+1} \frac{\lambda_{n} y}{\left(\gamma^{n}(y)-s\right)^{2}} \\
& \leqslant \frac{1}{s_{0}-s} \sum_{n=0}^{j+1} \frac{\lambda_{n} b_{j+2}}{\left(1-b_{i+2} / b_{n}\right)^{2}} \leqslant \frac{\lambda_{0} b_{0}}{s_{0}-s} \sum_{n=0}^{j+1} \rho^{n}(n+1)^{4}, \quad i=3,7,9 .
\end{aligned}
$$

Выберем теперь $b_{0} \in\left(0, T /\left(2 s_{0}\right)\right)$ таким, что

$$
\rho \leqslant 1, \quad \lambda_{0} b_{0} \sum_{n=0}^{\infty} \rho^{n}(n+1)^{6} \leqslant R .
$$

Тогда

$$
\begin{gathered}
\left\|\varphi_{1}^{j+2}-\varphi_{1}^{0}\right\|_{s}(y, t) \leqslant R ; \quad\left\|\varphi_{i}^{j+2}-\varphi_{i}^{0}\right\|_{s}(y) \leqslant R, \quad i=6,8 ; \quad\left\|\varphi_{5}^{j+2}-\varphi_{5}^{0}\right\|_{s}(y) \leqslant \frac{R}{\left(s_{0}-s\right)^{2}} \\
\left\|\varphi_{3}^{j+2}-\varphi_{3}^{0}\right\|_{s}(y, t) \leqslant \frac{R}{s_{0}-s} ; \quad\left\|\varphi_{i}^{j+2}-\varphi_{i}^{0}\right\|_{s}(y) \leqslant \frac{R}{s_{0}-s}, \quad i=7,9 ; \quad(y, t, s) \in F_{j+2} .
\end{gathered}
$$

Так как выбор $b_{0}$ не зависит от номера приближений, то последовательные приближения $\varphi_{i}^{n}, i=1,3,5,6,7,8,9$, принадлежат

$$
C_{(y, t)}\left(F ; A_{s}\right), \quad F=\bigcap_{n=0}^{\infty} F_{n}
$$

и для них имеют место неравенства

$$
\begin{gathered}
\left\|\varphi_{1}^{n}-\varphi_{1}^{0}\right\|_{s}(y, t) \leqslant R ; \quad\left\|\varphi_{i}^{n}-\varphi_{i}^{0}\right\|_{s}(y) \leqslant R, \quad i=6,8 ; \quad\left\|\varphi_{5}^{n}-\varphi_{5}^{0}\right\|_{s}(y) \leqslant \frac{R}{\left(s_{0}-s\right)^{2}} \\
\left\|\varphi_{3}^{n}-\varphi_{3}^{0}\right\|_{s}(y, t) \leqslant \frac{R}{s_{0}-s} ; \quad\left\|\varphi_{i}^{n}-\varphi_{i}^{0}\right\|_{s}(y) \leqslant \frac{R}{s_{0}-s}, \quad i=7,9 ; \quad(y, t, s) \in F .
\end{gathered}
$$

При $s \in\left(0, s_{0}\right)$ ряды

$$
\sum_{n=0}^{\infty}\left(\varphi_{i}^{n}-\varphi_{i}^{n-1}\right)
$$

сходятся равномерно в норме пространства

$$
C_{(y, t)}\left(P_{s T} ; A_{s}\right), \quad P_{s T}=G_{T} \cap\left\{(y, t): 0 \leqslant y \leqslant b\left(s_{0}-s\right)\right\},
$$


поэтому $\psi_{i}^{n} \rightarrow \psi_{i}$. Предельные функции $\psi_{i}$ являются элементами $C_{(y, t)}\left(P_{s T} ; A_{s}\right)$ и удовлетворяют уравнениям (2.36)-(2.42). Докажем теперь, что найденное решение единственно. Пусть $\psi_{i}$ и $\bar{\psi}_{i}$ - любые два решения, удовлетворяющие неравенствам

$$
\begin{gathered}
\left\|\varphi_{1}-\varphi_{1}^{0}\right\|_{s}(y, t) \leqslant R, \quad\left\|\varphi_{i}-\varphi_{i}^{0}\right\|_{s}(y) \leqslant R, \quad i=6,8, \quad\left\|\varphi_{5}-\varphi_{5}^{0}\right\|_{s}(y) \leqslant \frac{R}{\left(s_{0}-s\right)^{2}} \\
\left\|\varphi_{3}-\varphi_{3}^{0}\right\|_{s}(y, t) \leqslant \frac{R}{s_{0}-s}, \quad\left\|\varphi_{i}-\varphi_{i}^{0}\right\|_{s}(y) \leqslant \frac{R}{s_{0}-s}, \quad i=7,9, \quad(y, t, s) \in F
\end{gathered}
$$

Обозначим через $\tilde{\psi}_{i}=\varphi_{i}-\bar{\varphi}_{i}$ и пусть

$$
\begin{aligned}
\lambda:=\max \left\{\sup _{(y, t, s) \in F}\left[\left\|\tilde{\varphi}_{1}\right\|_{s}(y, t) \frac{\gamma(y)-s}{y}\right], \sup _{(y, t, s) \in F}\left[\left\|\tilde{\varphi}_{3}\right\|_{s}(y, t) \frac{(\gamma(y)-s)^{2}}{y}\right],\right. \\
\sup _{(y, t, s) \in F}\left[\left\|\tilde{\varphi}_{i}\right\|_{s}(y) \frac{\gamma(y)-s}{y}\right], i=6,8, \\
\left.\sup _{(y, t, s) \in F}\left[\left\|\tilde{\varphi}_{i}\right\|_{s}(y) \frac{(\gamma(y)-s)^{2}}{y}\right], i=7,9, \sup _{(y, t, s) \in F}\left[\left\|\tilde{\varphi}_{5}\right\|_{s}(y) \frac{(\gamma(y)-s)^{3}}{y}\right]\right\}<\infty,
\end{aligned}
$$

где $\gamma(y)=s_{0}-y / b, b=b_{0} \prod_{n=0}^{\infty}\left(1+1 /(n+1)^{2}\right)^{-1}$. Тогда для функций $\tilde{\varphi}_{i}$ можно получить соотношения

$$
\begin{aligned}
\tilde{\psi}_{1}(x, y, t) & =\frac{1}{2} \int_{0}^{y} \int_{t-y+\xi}^{t+y-\xi}\left\{H(x, \xi) \tilde{w}(x, \xi, \tau)-\frac{a}{2} \tilde{h}(x, \tau-\xi)+\tilde{k}_{00}(x, \xi, \tau)\right. \\
& -\int_{0}^{\tau-\xi}(\tilde{h}(x, \gamma) \bar{w}(x, \xi, \tau-\gamma)-h(x, \gamma) \tilde{w}(x, \xi, \tau-\gamma)) d \gamma \\
& +\nu^{2} \exp (r(x, 0) \tau / 2) \frac{\partial}{\partial x}\left\{L_{0}\left[\bar{\varphi}_{8}(x, \tau) \exp (-r(x, 0)(\tau) / 2),\left(\tilde{r}_{00}+\frac{\partial \tilde{\psi}_{2}}{\partial x}\right)(x, \xi, \tau)\right]\right. \\
& \left.\left.-\int_{0}^{\tau-\xi} \tilde{\psi}_{8}(x, \gamma) \exp (-r(x, 0) \gamma / 2)\left(r_{00}+\frac{\partial \varphi_{2}}{\partial x}\right)(x, \xi, \tau-\gamma) d \gamma\right\}\right\} d \tau d \xi \\
\tilde{\psi}_{3}(x, y, t) & =\frac{1}{2} \int_{-y}^{y}\left\{H(x, y-|\xi|) \tilde{w}^{n}(x, y-|\xi|, \xi+\tau)-\frac{a}{2}\left(\tilde{h}^{n}(x, \xi+t-y+|\xi|)\right.\right. \\
& +\tilde{k}_{00}^{n}(x, y-|\xi|, t+|\xi|)-\int_{0}^{t-y+\xi+|\xi|}(\tilde{h}(x, \gamma) \bar{w}(x, y-|\xi|, t+\xi-\gamma)
\end{aligned}
$$




$$
\begin{aligned}
& \left.-h(x, \gamma) \tilde{w}^{n}(x, y-|\xi|, t+\xi-\gamma)\right) d \gamma+\nu^{2} \exp (r(x, 0)(\xi+t) / 2) \\
& \times \frac{\partial}{\partial x}\left\{L_{0}\left[\bar{\varphi}_{8}(x, \xi+t) \exp (-r(x, 0)(\xi+t) / 2),\left(\tilde{r}_{00}^{n}+\frac{\partial \tilde{\psi}_{2}}{\partial x}\right)(x, y-|\xi|, \xi+t)\right]\right. \\
& \left.\left.-\int_{0}^{t-y+\xi+|\xi|} \tilde{\psi}_{8}(x, \gamma) \exp (-r(x, 0) \gamma / 2)\left(r_{00}+\frac{\partial \varphi_{2}}{\partial x}\right)(x, y-\xi, \xi+t-\gamma) d \gamma\right\}\right\} \operatorname{sgn}(\xi) d \xi, \\
& \left.\tilde{\psi}_{5}(x, y)=\int_{0}^{y}\left(\frac{2}{a} \tilde{\psi}_{8}(x, \xi) \tilde{g}_{0 t t}(x, y-\xi)-\tilde{h}(x, y-\xi)\right) \bar{\varphi}_{8}(x, \xi)+h(x, y-\xi) \tilde{\psi}_{8}(x, \xi)\right) d \xi \\
& +\frac{1}{a} \int_{0}^{y / 2}\left\{H(x, \xi) \tilde{w}_{t}(x, \xi, y-\xi)+\tilde{k}_{00 t}(x, \xi, y-\xi)-\frac{1}{2} \tilde{h}(x, y-2 \xi) \beta(x, \xi)\right. \\
& -\int_{0}^{y-2 \xi}\left(\tilde{h}(x, \tau) \bar{w}_{t}(x, \xi, y-\xi-\tau)-h(x, \tau) \tilde{w}_{t}(x, \xi, y-\xi-\tau)\right) d \tau \\
& +\nu^{2} \exp (r(x, 0)(y-\xi) / 2)\left\{\frac { \partial } { \partial x } \left\{L _ { 0 } \left[\bar{\varphi}_{8}(x, y-\xi) \exp (-r(x, 0)(y-\xi) / 2),\left(\tilde{r}_{00 t}^{n}+\frac{\partial \tilde{\psi}_{4}}{\partial x}\right)\right.\right.\right. \\
& \left.\times(x, \xi, y-\xi)]-\int_{0}^{y-2 \xi} \tilde{\psi}_{8}(x, \tau) \exp (-r(x, 0) \tau / 2)\left(r_{00 t}+\frac{\partial \varphi_{4}}{\partial x}\right)(x, \xi, y-\xi-\tau) d \tau\right\} \\
& +\frac{r(x, 0)}{2} \frac{\partial}{\partial x}\left\{L_{0}\left[\bar{\varphi}_{8}(x, \tau) \exp (-r(x, 0)(y-\xi) / 2),\left(\tilde{r}_{00}+\frac{\partial \tilde{\psi}_{2}}{\partial x}\right)(x, \xi, y-\xi)\right]\right. \\
& \left.-\int_{0}^{y-2 \xi} \tilde{\psi}_{8}(x, \gamma) \exp (-r(x, 0) \tau / 2)\left(r_{00}+\frac{\partial \varphi_{2}}{\partial x}\right)(x, \xi, y-\xi-\tau) d \gamma\right\} \\
& +\frac{1}{2} \frac{\partial}{\partial x}\left[\operatorname { e x p } ( - r ( x , 0 ) ( y - 2 \xi ) / 2 ) \left(\bar{\varphi}_{8}(x, y-2 \xi)\left(\tilde{r}_{00}+\frac{\partial \tilde{\psi}_{2}}{\partial x}\right)(x, \xi, \xi)\right.\right. \\
& \left.\left.\left.-\tilde{\psi}_{8}(x, y-2 \xi)\left(r_{00}+\frac{\partial \varphi_{2}}{\partial x}\right)(x, \xi, \xi)\right)\right]\right\} d \xi \\
& \tilde{\psi}_{6}(x, y)=\int_{0}^{y}(y-\xi)\left(\tilde{h}(x, \xi)+r(x, 0) \tilde{\psi}_{7}(x, \xi)-\frac{r^{2}(x, 0)}{4} \tilde{\psi}_{6}(x, \xi)\right) d \xi, \\
& \tilde{\psi}_{7}(x, y)=\int_{0}^{y}\left(\tilde{h}(x, \xi)+r(x, 0) \tilde{\psi}_{7}(x, \xi)-\frac{r^{2}(x, 0)}{4} \tilde{\psi}_{6}(x, \xi)\right) d \xi \\
& \tilde{\psi}_{8}(x, y)=\int_{0}^{y}(y-\xi)\left(c_{0}(x) \tilde{\psi}_{8}(x, \xi)-L\left[\varphi_{8}, \tilde{h}\right]-\int_{0}^{\xi} \tilde{\psi}_{8}(x, \xi-\tau) \bar{h}(x, \tau)\right) d \xi, \\
& \tilde{\psi}_{9}(x, y)=\int_{0}^{y}\left(c_{0}\left(\tilde{\psi}_{8}(x, \xi)-L\left[\varphi_{8}, \tilde{h}\right]-\int_{0}^{\xi} \tilde{\psi}_{8}(x, \xi-\tau) \bar{h}(x, \tau)\right) d \xi\right.
\end{aligned}
$$

где

$$
\begin{aligned}
\tilde{k}_{00}(x, y, t) & =\frac{\nu^{2} a}{2} \exp (k(x, 0)(t-y) / 2)\left[\left(k_{x x}(x, 0) \frac{y}{2}+k_{x}^{2}(x, 0) \frac{y^{2}}{4}+\frac{k_{x}^{2}(x, 0)(t-y)}{2}\right)\right. \\
& \left.\times \tilde{\psi}_{8}(x, t-y)+k_{x}(x, 0) \frac{\partial \tilde{\psi}_{8}}{\partial x}(x, t-y)\right],
\end{aligned}
$$




$$
\begin{aligned}
& \tilde{r}_{00}(x, y, t)=\frac{\partial}{\partial x}\left(\tilde{\psi}_{6}(x, t-y) \exp (k(x, 0) t / 2)\right), \\
& \tilde{k}_{00 t}(x, y, t)=\frac{k(x, 0)}{2} k_{00}(x, y, t)+\frac{\nu^{2} a}{2} \exp (k(x, 0)(t-y) / 2)\left[\left(k_{x x}(x, 0) \frac{y}{2}+k_{x}^{2}(x, 0) \frac{y^{2}}{4}\right.\right. \\
& \left.\left.+\frac{k_{x}^{2}(x, 0)(t-y)}{2}\right) \tilde{\psi}_{9}(x, t-y)+k_{x}(x, 0) \frac{\partial \tilde{\psi}_{9}}{\partial x}(x, t-y)+\frac{k_{x}^{2}(x, 0)}{2} \tilde{\psi}_{8}(x, t-y)\right], \\
& \tilde{r}_{00 t}(x, y, t)=\frac{\partial}{\partial x}\left[\exp (k(x, 0) t / 2)\left(\tilde{\psi}_{7}(x, t-y)+\frac{k(x, 0)}{2} \tilde{\psi}_{6}(x, t-y)\right)\right], \\
& \tilde{w}(x, y, t)=\tilde{\psi}_{1}(x, y, t)+\frac{1}{2}\left\{\frac{a}{2}\left(\tilde{\psi}_{8}(x, t+y)+\tilde{\psi}_{8}(x, t-y)\right)+\int_{0}^{t+y} \tilde{\psi}_{8}(x, \tau) \tilde{g}_{0}(x, t+y-\tau) d \tau\right. \\
& \left.+\int_{0}^{t-y} \tilde{\psi}_{8}(x, \tau) \tilde{g}_{0}(x, t-y-\tau) d \tau\right\} \\
& \tilde{w}_{t}(x, y, t)=\tilde{\psi}_{3}(x, y, t)+\frac{a}{4}\left[\tilde{\psi}_{9}(x, t+y)+\tilde{\psi}_{9}(x, t-y)\right]+\frac{1}{2} g_{0}(x, 0)\left[\tilde{\psi}_{8}(x, t+y)+\tilde{\psi}_{8}(x, t-y)\right] \\
& +\frac{1}{2}\left(\int_{0}^{t+y} \tilde{\psi}_{8}(x, t-\tau) \tilde{g}_{0 t}(x, \tau) d \tau+\int_{0}^{t-y} \tilde{\psi}_{8}(x, t-\tau) \tilde{g}_{0 t}(x, \tau) d \tau\right) \\
& \tilde{h}(x, y)=\frac{1}{2}\left[\tilde{\psi}_{5}(x, y)+c_{0}(x) \tilde{\psi}_{8}(x, y)\right]+\frac{1}{a}\left[g_{0}(x, 0) \tilde{\psi}_{9}(x, y)+\tilde{\psi}_{8}(x, y) \tilde{g}_{0 t}(x, 0)\right], \\
& \tilde{\psi}_{2}(x, y, t)=\exp (k(x, 0) t / 2)\left(L_{0}\left[\bar{\varphi}_{6}, \tilde{w}_{t}\right]-\int_{y}^{t} \tilde{\psi}_{6}(x, t-\tau) w(x, y, \tau)\right) d \tau, \\
& \tilde{\psi}_{4}(x, y, t)=\frac{k(x, 0)}{2} \tilde{\psi}_{2}(x, y, t)+\exp (k(x, 0) t / 2)\left(L_{0}\left[\bar{\varphi}_{6}(x, y), \tilde{w}_{t}(x, y, t)\right]-\tilde{\psi}_{6}(x, t-y) \beta(x, y)\right. \\
& \left.-\int_{y}^{t} \tilde{\psi}_{6}(x, y-\tau) w(x, y, \tau) d \tau\right) .
\end{aligned}
$$

Применяя к ним оценки, приведенные выше, находим неравенство

$$
\lambda \leqslant \lambda \rho^{\prime}, \quad \rho^{\prime}=\max \left(\mu_{5}^{\prime}, \mu_{6}^{\prime}, \mu_{7}^{\prime}, \max \left(1, b^{-1}\right) \mu_{8}^{\prime}, \max \left(1, b^{-1}\right) \mu_{9}^{\prime}\right)<\rho<1,
$$

где $\mu_{5,6,7}^{\prime}=\mu_{5,6,7}^{\prime}\left(b, a, R, s_{0}\right), \mu_{8,9}^{\prime}=\mu_{8,9}^{\prime}\left(b, a, R, s_{0}, T\right)$.

Следовательно, $\lambda=0$. Поэтому $\varphi_{i}=\bar{\varphi}_{i}$. Теорема доказана.

\section{Литература}

1. Туаева Ж. Д. Многомерная математическая модель сейсмики с памятью // Мат. форум. Т. 1, ч. 2. Исследования по диф. уравнениям и мат. моделированию.-Владикавказ: ВНЦ РАН, 2008.С. 297-306.-(Итоги науки. ЮФО).

2. Дурдиев Д. К., Тотиева Ж. Д. Задача об определении одномерного ядра уравнения вязкоупругости // Сиб. журн. индустр. матем.-2013.-Т. 16, № 2.-С. 72-82.

3. Овсянников Л. В. Сингулярный оператор в шкале банаховых пространств // Докл. АН CССР.1965.-Т. 163, вып. 4.-С. 819-822.

4. Овсянников Л. В. Нелинейная задача Коши в шкалах банаховых пространств // Докл. АН CCCP.-1971.-Т. 200, вып. 4.-С. 789-792. 
5. Nirenberg L. Topics in Nonlinear Functional Analysis.-N. Y.: Courant Institute Math. Sci., New York Univ., 1974.-259 p.

6. Романов В. Г. О локальной разрешимости некоторых многомерных обратных задач для уравнений гиперболического типа // Диф. уравнения.-1989.-Т. 25, № 2.-С. 275-284.

7. Романов В. Г. Вопросы корректности задачи определения скорости звука // Сиб. мат. журн.1989.-Т. 30, вып. 4.-С. 125-134.

8. Романов В. Г. О разрешимости обратных задач для гиперболических уравнений в классе функций, аналитических по части переменных // Докл. АН СССР.-1989.-Т. 304, вып. 4.-С. 807-811.

9. Дурдиев Д. К. Многомерная обратная задача для уравнения с памятью // Сиб. мат. журн.1994.-T. 35, вып. 3.-С. 574-582.

10. Durdiev $D$. K. Some multidimensional inverse problems of memory determination in hyperbolic equations // Zh. Mat. Fiz. Anal. Geom.-2007.-Vol. 3, № 4.-C. 411-423.

11. Дурдиев Д. К., Сафаров Ж. Ш. Локальная разрешимость задачи определения пространственной части многомерного ядра в интегродифференщиальном уравнении гиперболического типа // Вестн. Сам. гос. техн. ун-та. Сер. Физ.-мат. науки.-2012.-Т. 4, вып. 29.-С. 37-47.

Статъл поступила 9 февраля 2015 г.

ДурдиЕв ДурдимУРОд КАлАНдАРОВИч

Бухарский государственный университет, проректор по учебной работе

УЗБЕКИСТАН, 200117, Бухара, ул. М. Икбол, 11

E-mail: durdiev65@mail.ru

ТотиЕвА ЖАннА ДмитриЕвнА

Центр геофизических исследований ВНЦ РАН, старший научный сотрудник

РОССИЯ, 362002, Владикавказ, ул. Маркова, 93 а;

Северо-Осетинский государственный университет им. К. Л. Хетагурова, доцент кафедры математического анализа РОССИЯ, 362025, Владикавказ, ул. Ватутина, 46

E-mail: jannatuaeva@inbox.ru

\title{
THE PROBLEM OF DETERMINING THE MULTIDIMENSIONAL KERNEL OF VISCOELASTICITY EQUATION
}

\author{
Durdiev D. Q., Totieva Zh. D.
}

The integro-differential system of viscoelasticity equations is considered. The direct problem of determining of the displacements vector from the initial-boundary problem for this system is formulated. It is assumed that the kernel in the integral part depends on both the time and the space variable $x_{2}$. For its determination an additional condition relative to the first component of the displacements vector with $x_{3}=0$ is posed. The inverse problem is replaced by the equivalent system of integral equations. The study is based on the method of scales of Banach spaces of analytic functions. The theorem on local unique solvability of the inverse problem is proved in the class of functions analytic on the variable $x_{2}$ and continuous on $t$.

Key words: inverse problem, stability, delta function, Lame's coefficients, kernel. 